\title{
Uniqueness of positive solutions for several classes of sum operator equations and applications
}

\author{
Chen Yang ${ }^{1}$, Chengbo Zhai ${ }^{2 *}$ and Mengru Hao ${ }^{2}$
}

\section{"Correspondence:}

cbzhai@sxu.edu.cn;

cbzhai215@sohu.com

${ }^{2}$ School of Mathematical Sciences,

Shanxi University, Taiyuan, Shanxi 030006, P.R. China

Full list of author information is

available at the end of the article

\begin{abstract}
In this article we study several classes of sum operator equations on ordered Banach spaces and present some new existence and uniqueness results of positive solutions, which extend the existing corresponding results. Moreover, we establish some pleasant properties of nonlinear eigenvalue problems for several classes of sum operator equations. As applications, we utilize the main results obtained in this paper to study two classes nonlinear problems; one is the integral equation $u(t)=\lambda \int_{a}^{b} G(t, s) f(s, u(s)) d s$, where $f$ and $G$ are both nonnegative, $\lambda>0$ is a parameter; the other is the elliptic boundary value problem for the Lane-Emden-Fowler equation $-\Delta u=\lambda f(x, u), u(x)>0$ in $\Omega, u(x)=0$ on $\partial \Omega$, where $\Omega$ is a bounded domain with smooth boundary in $\mathbf{R}^{N}(N \geq 1), \lambda>0$ and $f(x, u)$ is allowed to be singular on $\partial \Omega$. The new results on the existence and uniqueness of positive solutions for these problems are given, which complement the existing results of positive solutions for these problems in the literature.
\end{abstract}

MSC: 47H10; 47H07;45G15; 35J60; 35J65

Keywords: positive solution; operator equation; normal cone; integral equation; Lane-Emden-Fowler equation

\section{Introduction and preliminaries}

With the development of nonlinear sciences, nonlinear functional analysis has been an active area of research over the past several decades. As an important branch of nonlinear functional analysis, nonlinear operator theory has attracted much attention and has been widely studied, especially nonlinear operators which arise in the connection with nonlinear differential and integral equations have been extensively studied (see for instance [1-12]). It is well known that the existence and uniqueness of positive solutions to nonlinear operator equations is very important in theory and applications. Many authors have studied this problem; for a small sample of such work, we refer the reader to $[7,10,13-23]$. The operator equation considered in this papers is always of the following form:

$$
A x=x \quad \text { or } A(x, x)=x .
$$

In [23], Zhao considered the existence of solutions for the sum operator equation

$$
A x+B x=x,
$$

@2014 Yang et al.; licensee Springer. This is an Open Access article distributed under the terms of the Creative Commons Attribution License (http://creativecommons.org/licenses/by/2.0), which permits unrestricted use, distribution, and reproduction in any medium, provided the original work is properly cited. 
where $A$ is increasing $e$-concave, $B$ is increasing $e$-convex and $A+B$ is a strict set contraction. Motivated by the works [22, 23], Sang et al. considered the operator equation (1.1), where $A$ is $\varphi_{1}$-concave, $B$ is $\varphi_{2}$-convex and $A+B$ is also a strict set contraction. However, we can see that the conditions of the main results in $[23,24]$ are strong and of utmost convenience.

Recently, we considered successively the operator equation (1.1) and the following operator equation:

$$
A(x, x)+B x=x,
$$

the operators $A, B$ in (1.1) are increasing, $\alpha$-concave and sub-homogeneous, respectively; the operators $A, B$ in (1.2) are mixed monotone and increasing $\alpha$-concave (or subhomogeneous), respectively. In [7], by using the properties of cones and a fixed point theorem for increasing general $\alpha$-concave operators, we established the existence and uniqueness of positive solutions for the operator equation (1.1), and we utilized the main results to present the existence and uniqueness of positive solutions for the following two problems; one is a fourth-order two-point boundary value problem for elastic beam equations,

$$
\left\{\begin{array}{l}
u^{\prime \prime \prime \prime}(t)=f(t, u(t)), \quad 0 \leq t \leq 1, \\
u(0)=u^{\prime}(0)=0, \\
u^{\prime \prime}(1)=0, \quad u^{\prime \prime \prime}(1)=g(u(1)),
\end{array}\right.
$$

where $f \in C([0,1] \times \mathbf{R})$ and $g \in C(\mathbf{R})$ are real functions; and the other is an elliptic value problem for Lane-Emden-Fowler equations

$$
\begin{cases}-\Delta u=f(x, u)+g(x, u), & x \in \Omega, \\ u(x)>0, & x \in \Omega, \\ u(x)=0, & x \in \partial \Omega,\end{cases}
$$

where $f(x, u), g(x, u)$ are allowed to be singular on $\partial \Omega$. In [21], by using the properties of cones and a fixed point theorem for mixed monotone operators, we established the existence and uniqueness of positive solutions for the operator equation (1.2), and we utilized the results obtained to study the existence and uniqueness of positive solutions for a nonlinear fractional differential equation boundary value problem,

$$
\left\{\begin{array}{l}
D_{0+}^{\alpha} u(t)=f(t, u(t), u(t))+g(t, u(t)), \quad 0<t<1, \\
u(0)=u(1)=u^{\prime}(0)=u^{\prime}(1)=0,
\end{array}\right.
$$

where $D_{0+}^{\alpha}$ is the Riemann-Liouville fractional derivative of order $\alpha>0$. These results are useful and interesting. For completeness, in this paper we will further consider the following several classes of sum operators:

(i) the sum of increasing operators and decreasing operators;

(ii) the sum of increasing operators and mixed monotone operators;

(iii) the sum of decreasing operators and mixed monotone operators;

(iv) the sum of increasing operators, decreasing operators and mixed monotone operators. 
Motivated by our works [7, 10, 21], we will study the above cases (i)-(iv). So this article is a continuation of our papers $[7,10,21]$, and we will present some interesting results on the existence and uniqueness of positive solutions for the above several classes of sum operator equations. To demonstrate the applicability of our abstract results, we give, in the last section of the paper, some applications to nonlinear integral equations and elliptic boundary value problems for the Lane-Emden-Fowler equations.

In the following two subsections, we state some definitions, notations, and known results. For convenience of the readers, we refer to [7-13, 20-22, 25-27] for details.

\subsection{Some basic definitions and notations}

Suppose that $E$ is a real Banach space which is partially ordered by a cone $P \subset E$, i.e., $x \leq y$ if and only if $y-x \in P$. If $x \leq y$ and $x \neq y$, then we denote $x<y$ or $y>x$. By $\theta$ we denote the zero element of $E$. Recall that a non-empty closed convex set $P \subset E$ is a cone if it satisfies (i) $x \in P, \lambda \geq 0 \Rightarrow \lambda x \in P$; (ii) $x \in P,-x \in P \Rightarrow x=\theta$.

Putting $\stackrel{\circ}{P}=\{x \in P \mid x$ is an interior point of $P\}$, a cone $P$ is said to be solid if $\stackrel{\circ}{P}$ is nonempty. Moreover, $P$ is called normal if there exists a constant $N>0$ such that, for all $x, y \in$ $E, \theta \leq x \leq y$ implies $\|x\| \leq N\|y\|$; in this case $N$ is called the normality constant of $P$. If $x_{1}, x_{2} \in E$, the set $\left[x_{1}, x_{2}\right]=\left\{x \in E \mid x_{1} \leq x \leq x_{2}\right\}$ is called the order interval between $x_{1}$ and $x_{2}$. We say that an operator $A: E \rightarrow E$ is increasing (decreasing) if $x \leq y$ implies $A x \leq A y$ $(A x \geq A y)$.

For all $x, y \in E$, the notation $x \sim y$ means that there exist $\lambda>0$ and $\mu>0$ such that $\lambda x \leq y \leq \mu x$. Clearly, $\sim$ is an equivalence relation. Given $h>\theta$ (i.e., $h \geq \theta$ and $h \neq \theta$ ), we denote by $P_{h}$ the set $P_{h}=\{x \in E \mid x \sim h\}$. It is easy to see that $P_{h} \subset P$.

Definition 1.1 Let $D=P$ or $D=\stackrel{\circ}{P}$ and $\alpha$ be a real number with $0 \leq \alpha<1$. An operator $A: P \rightarrow P$ is said to be $\alpha$-concave if it satisfies

$$
A(t x) \geq t^{\alpha} A x, \quad \forall t \in(0,1), x \in D .
$$

Notice that the definition of an $\alpha$-concave operator mentioned above is different from that in [26], because we need not require the cone to be solid in general.

Definition 1.2 An operator $A: P \rightarrow P$ is said to be sub-homogeneous if it satisfies

$$
A(t x) \geq t A x, \quad \forall t \in(0,1), x \in P .
$$

Definition 1.3 (See $[10,21,27]) A: P \times P \rightarrow P$ is said to be a mixed monotone operator if $A(x, y)$ is increasing in $x$ and decreasing in $y$, i.e., $u_{i}, v_{i}(i=1,2) \in P, u_{1} \leq u_{2}, v_{1} \geq v_{2}$ imply $A\left(u_{1}, v_{1}\right) \leq A\left(u_{2}, v_{2}\right)$. An element $x \in P$ is called a fixed point of $A$ if $A(x, x)=x$.

\subsection{Some fixed point theorems and properties}

In this subsection, we assume that $E$ is a real Banach space with a partial order introduced by a cone $P$ of $E$. Take $h \in E, h>\theta, P_{h}$ is given as in Section 1.1.

In the paper [7], we considered the existence and uniqueness of positive solutions to the operator equation (1.1) on ordered Banach spaces and established the following conclusion. 
Theorem 1.1 (See Theorem 2.2 in [7]) Let $P$ be a normal cone in $E, A: P \rightarrow P$ be an increasing $\alpha$-concave operator and $B: P \rightarrow P$ be an increasing sub-homogeneous operator. Assume that

(i) there is $h>\theta$ such that $A h \in P_{h}$ and $B h \in P_{h}$;

(ii) there exists a constant $\delta_{0}>0$ such that $A x \geq \delta_{0} B x, \forall x \in P$.

Then the operator equation (1.1) has a unique solution $x^{*}$ in $P_{h}$. Moreover, constructing successively the sequence $y_{n}=A y_{n-1}+B y_{n-1}, n=1,2, \ldots$ for any initial value $y_{0} \in P_{h}$, we have $y_{n} \rightarrow x^{*}$ as $n \rightarrow \infty$.

In the paper [10], we present the following fixed point theorem for a class of general mixed monotone operators and established some pleasant properties of nonlinear eigenvalue problems for mixed monotone operators.

Theorem 1.2 (See Lemma 2.1 and Theorem 2.1 in [10]) Let $P$ be a normal cone in $E$. Assume that $A: P \times P \rightarrow P$ is a mixed monotone operator and satisfies:

(i) there exists $h \in P$ with $h \neq \theta$ such that $A(h, h) \in P_{h}$;

(ii) for any $u, v \in P$ and $t \in(0,1)$, there exists $\varphi(t) \in(t, 1]$ such that $A\left(t u, t^{-1} v\right) \geq \varphi(t) A(u, v)$.

Then:

(1) $T: P_{h} \times P_{h} \rightarrow P_{h}$;

(2) there exist $u_{0}, v_{0} \in P_{h}$ and $r \in(0,1)$ such that $r v_{0} \leq u_{0}<v_{0}$, $u_{0} \leq A\left(u_{0}, v_{0}\right) \leq A\left(v_{0}, u_{0}\right) \leq v_{0}$

(3) the operator equation (1.2) has a unique solution $x^{*}$ in $P_{h}$;

(4) for any initial values $x_{0}, y_{0} \in P_{h}$, constructing successively the sequences

$$
x_{n}=A\left(x_{n-1}, y_{n-1}\right), \quad y_{n}=A\left(y_{n-1}, x_{n-1}\right), \quad n=1,2, \ldots,
$$

we have $x_{n} \rightarrow x^{*}$ and $y_{n} \rightarrow x^{*}$ as $n \rightarrow \infty$.

Theorem 1.3 (See Theorem 2.3 in [10]) Assume that the operator A satisfies the conditions of Theorem 1.2. Let $x_{\lambda}(\lambda>0)$ denote the unique solution of nonlinear eigenvalue equation $A(x, x)=\lambda x$ in $P_{h}$. Then we have the following conclusions:

$\left(\mathrm{R}_{1}\right)$ If $\varphi(t)>t^{\frac{1}{2}}$ for $t \in(0,1)$, then $x_{\lambda}$ is strictly decreasing in $\lambda$, that is, $0<\lambda_{1}<\lambda_{2}$ implies $x_{\lambda_{1}}>x_{\lambda_{2}}$

$\left(\mathrm{R}_{2}\right)$ If there exists $\beta \in(0,1)$ such that $\varphi(t) \geq t^{\beta}$ for $t \in(0,1)$, then $x_{\lambda}$ is continuous in $\lambda$, that is, $\lambda \rightarrow \lambda_{0}\left(\lambda_{0}>0\right)$ implies $\left\|x_{\lambda}-x_{\lambda_{0}}\right\| \rightarrow 0$;

$\left(\mathrm{R}_{3}\right)$ If there exists $\beta \in\left(0, \frac{1}{2}\right)$ such that $\varphi(t) \geq t^{\beta}$ for $t \in(0,1)$, then $\lim _{\lambda \rightarrow \infty}\left\|x_{\lambda}\right\|=0$, $\lim _{\lambda \rightarrow 0^{+}}\left\|x_{\lambda}\right\|=\infty$.

Based on Theorem 1.2, in [21] we considered the operator equation (1.2) and established the following conclusions.

Theorem 1.4 (See Theorem 2.1 in [21]) Let $P$ be a normal cone in $E, \alpha \in(0,1) . A: P \times P \rightarrow$ $P$ is a mixed monotone operator and satisfies

$$
A\left(t x, t^{-1} y\right) \geq t^{\alpha} A(x, y), \quad t \in(0,1), x, y \in P .
$$


$B: P \rightarrow P$ is an increasing sub-homogeneous operator. Assume that

(i) there is $h_{0} \in P_{h}$ such that $A\left(h_{0}, h_{0}\right) \in P_{h}$ and $B h_{0} \in P_{h}$;

(ii) there exists a constant $\delta_{0}>0$ such that $A(x, y) \geq \delta_{0} B x, \forall x, y \in P$.

Then:

(1) $A: P_{h} \times P_{h} \rightarrow P_{h}, B: P_{h} \rightarrow P_{h}$;

(2) there exist $u_{0}, v_{0} \in P_{h}$ and $r \in(0,1)$ such that

$$
r v_{0} \leq u_{0}<v_{0}, \quad u_{0} \leq A\left(u_{0}, v_{0}\right)+B u_{0} \leq A\left(v_{0}, u_{0}\right)+B v_{0} \leq v_{0} ;
$$

(3) the operator equation (1.2) has a unique solution $x^{*}$ in $P_{h}$;

(4) for any initial values $x_{0}, y_{0} \in P_{h}$, constructing successively the sequences

$$
x_{n}=A\left(x_{n-1}, y_{n-1}\right)+B x_{n-1}, \quad y_{n}=A\left(y_{n-1}, x_{n-1}\right)+B y_{n-1}, \quad n=1,2, \ldots,
$$

we have $x_{n} \rightarrow x^{*}$ and $y_{n} \rightarrow x^{*}$ as $n \rightarrow \infty$.

Theorem 1.5 (See Theorem 2.4 in [21]) Let P be a normal cone in $E, \alpha \in(0,1) . A: P \times P \rightarrow$ $P$ is a mixed monotone operator and satisfies

$$
A\left(t x, t^{-1} y\right) \geq t A(x, y), \quad t \in(0,1), x, y \in P .
$$

$B: P \rightarrow P$ is an increasing $\alpha$-concave operator. Assume that

(i) there is $h_{0} \in P_{h}$ such that $A\left(h_{0}, h_{0}\right) \in P_{h}$ and $B h_{0} \in P_{h}$;

(ii) there exists a constant $\delta_{0}>0$ such that $A(x, y) \leq \delta_{0} B x, \forall x, y \in P$.

Then:

(1) $A: P_{h} \times P_{h} \rightarrow P_{h}, B: P_{h} \rightarrow P_{h}$;

(2) there exist $u_{0}, v_{0} \in P_{h}$ and $r \in(0,1)$ such that

$$
r v_{0} \leq u_{0}<v_{0}, \quad u_{0} \leq A\left(u_{0}, v_{0}\right)+B u_{0} \leq A\left(v_{0}, u_{0}\right)+B v_{0} \leq v_{0} ;
$$

(3) the operator equation (1.2) has a unique solution $x^{*}$ in $P_{h}$;

(4) for any initial values $x_{0}, y_{0} \in P_{h}$, constructing successively the sequences

$$
x_{n}=A\left(x_{n-1}, y_{n-1}\right)+B x_{n-1}, \quad y_{n}=A\left(y_{n-1}, x_{n-1}\right)+B y_{n-1}, \quad n=1,2, \ldots,
$$

we have $x_{n} \rightarrow x^{*}$ and $y_{n} \rightarrow x^{*}$ as $n \rightarrow \infty$.

\section{Main results}

In this section we consider the existence and uniqueness of positive solutions for several classes of sum operator equations. We always assume that $E$ is a real Banach space with a partial order induced by a cone $P$ of $E$. Take $h \in E, h>\theta$ and $P_{h}$ as given in the Introduction.

\subsection{The sum of increasing operators and decreasing operators}

Now we first consider the following sum operator equations:

$$
\begin{aligned}
& A x+B x=x, \\
& A x+B x=\lambda x, \quad \lambda>0 .
\end{aligned}
$$


Theorem 2.1 Let $P$ be a normal cone, $A: P \rightarrow P$ be an increasing operator and $B: P \rightarrow P$ be a decreasing operator. Assume that:

$\left(\mathrm{H}_{11}\right)$ for any $x \in P$ and $t \in(0,1)$, there exist $\varphi_{i}(t) \in(t, 1)(i=1,2)$ such that

$$
A(t x) \geq \varphi_{1}(t) A x, \quad B(t x) \leq \frac{1}{\varphi_{2}(t)} B x
$$

$\left(\mathrm{H}_{12}\right)$ there exists $h_{0} \in P_{h}$ such that $A h_{0}+B h_{0} \in P_{h}$.

Then:

(i) there exist $u_{0}, v_{0} \in P_{h}$ and $r \in(0,1)$ such that

$$
r v_{0} \leq u_{0}<v_{0}, \quad u_{0} \leq A u_{0}+B v_{0} \leq A v_{0}+B u_{0} \leq v_{0}
$$

(ii) the operator equation (2.1) has a unique solution $x^{*}$ in $P_{h}$;

(iii) for any initial values $x_{0}, y_{0} \in P_{h}$, constructing successively the sequences

$$
x_{n}=A x_{n-1}+B y_{n-1}, \quad y_{n}=A y_{n-1}+B x_{n-1}, \quad n=1,2, \ldots,
$$

we have $x_{n} \rightarrow x^{*}, y_{n} \rightarrow x^{*}$ as $n \rightarrow \infty$.

Proof Firstly, from (2.3), we have

$$
A\left(\frac{1}{t} x\right) \leq \frac{1}{\varphi_{1}(t)} A x, \quad B\left(\frac{1}{t} x\right) \geq \varphi_{2}(t) B x, \quad \forall x \in P, t \in(0,1) .
$$

Since $A h_{0}+B h_{0} \in P_{h}$, there exist constants $\lambda_{1}, \lambda_{2}>0$ such that

$$
\lambda_{1} h \leq A h_{0}+B h_{0} \leq \lambda_{2} h .
$$

Also from $h_{0} \in P_{h}$, there exists a constant $t_{0} \in(0,1)$ such that

$$
t_{0} h \leq h_{0} \leq \frac{1}{t_{0}} h
$$

Let $\varphi(t)=\min \left\{\varphi_{1}(t), \varphi_{2}(t)\right\}, t \in(0,1)$. Then $\varphi(t) \in(t, 1)$. From (2.3) and (2.4), we obtain

$$
\begin{aligned}
A h+B h & \geq A\left(t_{0} h_{0}\right)+B\left(\frac{1}{t_{0}} h_{0}\right) \geq \varphi_{1}\left(t_{0}\right) A h_{0}+\varphi_{2}\left(t_{0}\right) B h_{0} \\
& \geq \varphi\left(t_{0}\right)\left(A h_{0}+B h_{0}\right) \geq \lambda_{1} \varphi\left(t_{0}\right) h, \\
A h+B h & \leq A\left(\frac{1}{t_{0}} h_{0}\right)+B\left(t_{0} h_{0}\right) \leq \frac{1}{\varphi_{1}\left(t_{0}\right)} A h_{0}+\frac{1}{\varphi_{2}\left(t_{0}\right)} B h_{0} \\
& \leq \frac{1}{\varphi\left(t_{0}\right)}\left(A h_{0}+B h_{0}\right) \leq \frac{\lambda_{2}}{\varphi\left(t_{0}\right)} h .
\end{aligned}
$$

Note that $\lambda_{1} \varphi\left(t_{0}\right), \frac{\lambda_{2}}{\varphi\left(t_{0}\right)}>0$, we can get $A h+B h \in P_{h}$.

Next we define an operator $T=A+B$ by $T(x, y)=A x+B y$. Then $T: P \times P \rightarrow P$ is a mixed monotone operator and $T(h, h)=A h+B h \in P_{h}$. Moreover, for any $x, y \in P$ and $t \in(0,1)$, 
we have

$$
T\left(t x, t^{-1} y\right)=A(t x)+B\left(t^{-1} y\right) \geq \varphi_{1}(t) A x+\varphi_{2}(t) B y \geq \varphi(t)(A x+B y)=\varphi(t) T(x, y) .
$$

Hence, the operator $T$ satisfies the condition (ii) in Theorem 1.2. An application of Theorem 1.2 implies that: there are $u_{0}, v_{0} \in P_{h}$ and $r \in(0,1)$ such that $r v_{0} \leq u_{0}<v_{0}, u_{0} \leq$ $T\left(u_{0}, v_{0}\right) \leq T\left(v_{0}, u_{0}\right) \leq v_{0}$; operator equation $T(x)=x$ has a unique positive $x^{*} \in P_{h}$; for any initial values $x_{0}, y_{0} \in P_{h}$, constructing successively the sequences

$$
x_{n}=T\left(x_{n-1}, y_{n-1}\right), \quad y_{n}=T\left(y_{n-1}, x_{n-1}\right), \quad n=1,2, \ldots,
$$

we have $\left\|x_{n}-x^{*}\right\| \rightarrow 0$ and $\left\|y_{n}-x^{*}\right\| \rightarrow 0$ as $n \rightarrow \infty$. That is:

(i) there exist $u_{0}, v_{0} \in P_{h}$ and $r \in(0,1)$ such that

$$
r v_{0} \leq u_{0}<v_{0}, \quad u_{0} \leq A u_{0}+B v_{0} \leq A v_{0}+B u_{0} \leq v_{0}
$$

(ii) the operator equation (2.1) has a unique solution $x^{*}$ in $P_{h}$;

(iii) for any initial values $x_{0}, y_{0} \in P_{h}$, constructing successively the sequences

$$
x_{n}=A x_{n-1}+B y_{n-1}, \quad y_{n}=A y_{n-1}+B x_{n-1}, \quad n=1,2, \ldots,
$$

we have $x_{n} \rightarrow x^{*}, y_{n} \rightarrow x^{*}$ as $n \rightarrow \infty$.

Note that $h \in P_{h}$, and we can easily obtain the following conclusions.

Corollary 2.2 Let $P$ be a normal cone, $A: P_{h} \rightarrow P_{h}$ be an increasing operator and $B: P_{h} \rightarrow$ $P_{h}$ be a decreasing operator. Assume that:

$\left(\mathrm{H}_{13}\right)$ for any $x \in P_{h}$ and $t \in(0,1)$, there exist $\varphi_{i}(t) \in(t, 1)(i=1,2)$ such that

$$
A(t x) \geq \varphi_{1}(t) A x, \quad B(t x) \leq \frac{1}{\varphi_{2}(t)} B x .
$$

Then:

(i) there exist $u_{0}, v_{0} \in P_{h}$ and $r \in(0,1)$ such that

$$
r v_{0} \leq u_{0}<v_{0}, \quad u_{0} \leq A u_{0}+B v_{0} \leq A v_{0}+B u_{0} \leq v_{0}
$$

(ii) the operator equation (2.1) has a unique solution $x^{*}$ in $P_{h}$;

(iii) for any initial values $x_{0}, y_{0} \in P_{h}$, constructing successively the sequences

$$
x_{n}=A x_{n-1}+B y_{n-1}, \quad y_{n}=A y_{n-1}+B x_{n-1}, \quad n=1,2, \ldots,
$$

we have $x_{n} \rightarrow x^{*}, y_{n} \rightarrow x^{*}$ as $n \rightarrow \infty$.

Corollary 2.3 Let $\alpha_{1}, \alpha_{2} \in(0,1)$. Let $P$ be a normal cone, $A: P \rightarrow P$ be an increasing $\alpha_{1}$-concave operator and $B: P \rightarrow P$ be a decreasing $\alpha_{2}$-convex operator. Assume that $\left(\mathrm{H}_{12}\right)$ holds. Then: 
(i) there exist $u_{0}, v_{0} \in P_{h}$ and $r \in(0,1)$ such that

$$
r v_{0} \leq u_{0}<v_{0}, \quad u_{0} \leq A u_{0}+B v_{0} \leq A v_{0}+B u_{0} \leq v_{0}
$$

(ii) the operator equation (2.1) has a unique solution $x^{*}$ in $P_{h}$;

(iii) for any initial values $x_{0}, y_{0} \in P_{h}$, constructing successively the sequences

$$
x_{n}=A x_{n-1}+B y_{n-1}, \quad y_{n}=A y_{n-1}+B x_{n-1}, \quad n=1,2, \ldots,
$$

we have $x_{n} \rightarrow x^{*}, y_{n} \rightarrow x^{*}$ as $n \rightarrow \infty$.

Proof Let $\varphi_{1}(t)=t^{\alpha_{1}}, \varphi_{2}(t)=t^{\alpha_{2}}, t \in(0,1)$. Then $\varphi_{1}(t), \varphi_{2}(t) \in(t, 1)$ for $t \in(0,1)$ and

$$
A(t x) \geq t^{\alpha_{1}} A x=\varphi_{1}(t) A x, \quad B(t x) \leq t^{-\alpha_{2}} B x=\frac{1}{\varphi_{2}(t)} B x, \quad x \in P .
$$

Hence, the conclusions follow from Theorem 2.1.

Corollary 2.4 Let $\alpha \in(0,1)$ and $P$ be a normal cone. Let $A_{1}: P \rightarrow P$ be an increasing $\alpha$-concave operator and $A_{2}: P \rightarrow P$ be an increasing sub-homogeneous operator, $B: P \rightarrow P$ be a decreasing operator which satisfies (2.3). Assume that:

$\left(\mathrm{H}_{14}\right)$ there exists $h_{0} \in P_{h}$ such that $A_{1} h_{0}+A_{2} h_{0} \in P_{h}$;

$\left(\mathrm{H}_{15}\right)$ there exists $\delta>0$ such that $A_{1} x \geq \delta A_{2} x, x \in P$;

$\left(\mathrm{H}_{16}\right)$ there exists $h_{1} \in P_{h}$ such that $B h_{1} \in P_{h}$.

Then:

(i) there exist $u_{0}, v_{0} \in P_{h}$ and $r \in(0,1)$ such that

$$
r v_{0} \leq u_{0}<v_{0}, \quad u_{0} \leq A_{1} u_{0}+A_{2} u_{0}+B v_{0} \leq A_{1} v_{0}+A_{2} v_{0}+B u_{0} \leq v_{0}
$$

(ii) the following operator equation:

$$
A_{1} x+A_{2} x+B x=x
$$

has a unique solution $x^{*}$ in $P_{h}$;

(iii) for any initial values $x_{0}, y_{0} \in P_{h}$, constructing successively the sequences

$$
\begin{aligned}
& x_{n}=A_{1} x_{n-1}+A_{2} x_{n-1}+B y_{n-1}, \\
& y_{n}=A_{1} y_{n-1}+A_{2} y_{n-1}+B x_{n-1}, \quad n=1,2, \ldots,
\end{aligned}
$$

we have $x_{n} \rightarrow x^{*}, y_{n} \rightarrow x^{*}$ as $n \rightarrow \infty$.

Proof Define an operator $A=A_{1}+A_{2}$ by $A x=A_{1} x+A_{2} x$. Then $A: P \rightarrow P$ is an increasing operator and $A h_{0} \in P_{h}$. Since $h_{0}, h_{1} \in P_{h}$, there exist $t_{0}, t_{1} \in(0,1)$ such that

$$
t_{0} h \leq h_{0} \leq \frac{1}{t_{0}} h, \quad t_{1} h \leq h_{1} \leq \frac{1}{t_{1}} h .
$$


Then $h_{0} \leq \frac{1}{t_{0}} h \leq \frac{1}{t_{0} t_{1}} h_{1}, h_{0} \geq t_{0} h \geq t_{0} t_{1} h_{1}$, and thus

$$
B h_{0} \geq B\left(\frac{1}{t_{0} t_{1}} h_{1}\right) \geq \varphi_{2}\left(t_{0} t_{1}\right) B h_{1}, \quad B h_{0} \leq B\left(t_{0} t_{1} h_{1}\right) \leq \frac{1}{\varphi_{2}\left(t_{0} t_{1}\right)} B h_{1} .
$$

Note that $\varphi_{2}\left(t_{0} t_{1}\right), \frac{1}{\varphi_{2}\left(t_{0} t_{1}\right)}>0$ and $B h_{1} \in P_{h}$, we can get $B h_{0} \in P_{h}$. Hence, $A h_{0}+B h_{0} \in P_{h}$.

From the proof of Theorem 1.1, there exists $\beta_{0}(t) \in(\alpha, 1)$ with respect to $t$, such that

$$
A(t x) \geq t^{\beta_{0}(t)} A x, \quad \forall t \in(0,1), x \in P .
$$

Let $\varphi_{1}(t)=t^{\beta_{0}(t)}, t \in(0,1)$. Then $\varphi_{1}(t) \in(t, 1)$ and $A(t x) \geq \varphi_{1}(t) A x, x \in P$.

Therefore, operators $A, B$ satisfy all the conditions of Theorem 2.1. So we easily obtain the following conclusions:

(i) there exist $u_{0}, v_{0} \in P_{h}$, and $r \in(0,1)$ such that

$$
r v_{0} \leq u_{0}<v_{0}, \quad u_{0} \leq A_{1} u_{0}+A_{2} u_{0}+B v_{0} \leq A_{1} v_{0}+A_{2} v_{0}+B u_{0} \leq v_{0}
$$

(ii) the operator equation (2.5) has a unique solution $x^{*}$ in $P_{h}$;

(iii) for any initial values $x_{0}, y_{0} \in P_{h}$, constructing successively the sequences

$$
\begin{aligned}
& x_{n}=A_{1} x_{n-1}+A_{2} x_{n-1}+B y_{n-1}, \\
& y_{n}=A_{1} y_{n-1}+A_{2} y_{n-1}+B x_{n-1}, \quad n=1,2, \ldots,
\end{aligned}
$$

we have $x_{n} \rightarrow x^{*}, y_{n} \rightarrow x^{*}$ as $n \rightarrow \infty$.

Corollary 2.5 Assume that all the conditions of Theorem 2.1 hold. Let $x_{\lambda}(\lambda>0)$ denote the unique solution of operator equation (2.2). Then we have the following conclusions:

(i) if $\varphi_{i}(t)>t^{\frac{1}{2}}(i=1,2)$ for $t \in(0,1)$, then $x_{\lambda}$ is strictly decreasing in $\lambda$, that is, $0<\lambda_{1}<\lambda_{2}$ implies $x_{\lambda_{1}}>x_{\lambda_{2}}$

(ii) if there exists $\beta \in(0,1)$ such that $\varphi_{i}(t) \geq t^{\beta}(i=1,2)$ for $t \in(0,1)$, then $x_{\lambda}$ is continuous in $\lambda$, that is, $\lambda \rightarrow \lambda_{0}\left(\lambda_{0}>0\right)$ implies $\left\|x_{\lambda}-x_{\lambda_{0}}\right\| \rightarrow 0$;

(iii) if there exists $\beta \in\left(0, \frac{1}{2}\right)$ such that $\varphi_{i}(t) \geq t^{\beta}(i=1,2)$ for $t \in(0,1)$, then $\lim _{\lambda \rightarrow \infty}\left\|x_{\lambda}\right\|=0, \lim _{\lambda \rightarrow 0^{+}}\left\|x_{\lambda}\right\|=\infty$.

Proof Define an operator $T=A+B$ by $T(x, y)=A x+B y$. Then $T: P \times P \rightarrow P$ is a mixed monotone operator. From the proof of Theorem 2.1, we have $T(h, h) \in P_{h}$, and

$$
T\left(t x, t^{-1} y\right) \geq \varphi(t) T(x, y), \quad t \in(0,1), x, y \in P,
$$

where $\varphi(t)=\min \left\{\varphi_{1}(t), \varphi_{2}(t)\right\}$. Evidently, $\varphi(t) \in(t, 1)$ for $t \in(0,1)$. Hence, the conclusions follow from Theorem 1.3.

Similarly, we can easily obtain the following result.

Corollary 2.6 Assume that all the conditions of Corollary 2.3 hold. Let $x_{\lambda}(\lambda>0)$ denote the unique solution of operator equation (2.2). Then we have the following conclusions: 
(i) if $\alpha_{1}, \alpha_{2} \in\left(0, \frac{1}{2}\right)$, then $x_{\lambda}$ is strictly decreasing in $\lambda$, that is, $0<\lambda_{1}<\lambda_{2}$ implies $x_{\lambda_{1}}>x_{\lambda_{2}}$

(ii) $x_{\lambda}$ is continuous in $\lambda$, that is, $\lambda \rightarrow \lambda_{0}\left(\lambda_{0}>0\right)$ implies $\left\|x_{\lambda}-x_{\lambda_{0}}\right\| \rightarrow 0$;

(iii) if $\alpha_{1}, \alpha_{2} \in\left(0, \frac{1}{2}\right)$, then $\lim _{\lambda \rightarrow \infty}\left\|x_{\lambda}\right\|=0, \lim _{\lambda \rightarrow 0^{+}}\left\|x_{\lambda}\right\|=\infty$.

\subsection{The sum of increasing operators and mixed monotone operators}

Next, we consider the following sum operator equations:

$$
\begin{aligned}
& A x+B(x, x)=x, \\
& A x+B(x, x)=\lambda x, \quad \lambda>0 .
\end{aligned}
$$

Theorem 2.7 Let $P$ be a normal cone, $A: P \rightarrow P$ be an increasing operator and $B: P \times P \rightarrow$ $P$ be a mixed monotone operator. Assume that:

$\left(\mathrm{H}_{21}\right)$ for any $x \in P, t \in(0,1)$, there exists $\varphi_{1}(t) \in(t, 1)$ such that

$$
A(t x) \geq \varphi_{1}(t) A x
$$

$\left(\mathrm{H}_{22}\right)$ for any $x, y \in P, t \in(0,1)$, there exists $\varphi_{2}(t) \in(t, 1)$ such that

$$
B\left(t x, t^{-1} y\right) \geq \varphi_{2}(t) B(x, y)
$$

$\left(\mathrm{H}_{23}\right)$ there exists $h_{0} \in P_{h}$ such that $A h_{0}+B\left(h_{0}, h_{0}\right) \in P_{h}$.

Then:

(i) there exist $u_{0}, v_{0} \in P_{h}$ and $r \in(0,1)$ such that

$$
r v_{0} \leq u_{0}<v_{0}, \quad u_{0} \leq A u_{0}+B\left(u_{0}, v_{0}\right) \leq A v_{0}+B\left(v_{0}, u_{0}\right) \leq v_{0} ;
$$

(ii) the operator equation (2.6) has a unique solution $x^{*}$ in $P_{h}$;

(iii) for any initial values $x_{0}, y_{0} \in P_{h}$, constructing successively the sequences

$$
x_{n}=A x_{n-1}+B\left(x_{n-1}, y_{n-1}\right), \quad y_{n}=A y_{n-1}+B\left(y_{n-1}, x_{n-1}\right), \quad n=1,2, \ldots,
$$

we have $x_{n} \rightarrow x^{*}, y_{n} \rightarrow x^{*}$ as $n \rightarrow \infty$.

Proof From (2.9), we obtain

$$
B\left(t^{-1} x, t y\right) \leq \frac{1}{\varphi_{2}(t)} B(x, y), \quad x, y \in P, t \in(0,1) .
$$

Since $A h_{0}+B\left(h_{0}, h_{0}\right) \in P_{h}$, there exist constants $\lambda_{1}, \lambda_{2}>0$ such that

$$
\lambda_{1} h \leq A h_{0}+B\left(h_{0}, h_{0}\right) \leq \lambda_{2} h
$$

Also, from $h_{0} \in P_{h}$, there exists a small constant $t_{0} \in(0,1)$ such that

$$
t_{0} h \leq h_{0} \leq \frac{1}{t_{0}} h
$$


Let $\varphi(t)=\min \left\{\varphi_{1}(t), \varphi_{2}(t)\right\}$. Then $\varphi(t) \in(t, 1)$ for $t \in(0,1)$. From (2.8)-(2.10),

$$
\begin{aligned}
A h+B(h, h) & \geq A\left(t_{0} h_{0}\right)+B\left(t_{0} h_{0}, t_{0}^{-1} h_{0}\right) \geq \varphi_{1}\left(t_{0}\right) A h_{0}+\varphi_{2}\left(t_{0}\right) B\left(h_{0}, h_{0}\right) \\
& \geq \varphi\left(t_{0}\right)\left[A h_{0}+B\left(h_{0}, h_{0}\right)\right] \geq \lambda_{1} \varphi\left(t_{0}\right) h, \\
A h+B(h, h) & \leq A\left(t_{0}^{-1} h_{0}\right)+B\left(t_{0}^{-1} h_{0}, t_{0} h_{0}\right) \leq \frac{1}{\varphi_{1}\left(t_{0}\right)} A h_{0}+\frac{1}{\varphi_{2}\left(t_{0}\right)} B\left(h_{0}, h_{0}\right) \\
& \leq \frac{1}{\varphi\left(t_{0}\right)}\left[A h_{0}+B\left(h_{0}, h_{0}\right)\right] \leq \frac{\lambda_{2}}{\varphi\left(t_{0}\right)} h .
\end{aligned}
$$

Note that $\lambda_{1} \varphi\left(t_{0}\right), \frac{\lambda_{2}}{\varphi\left(t_{0}\right)}>0$, we can get $A h+B(h, h) \in P_{h}$.

Next, we define an operator $T=A+B$ by $T(x, y)=A x+B(x, y)$. Then $T: P \times P \rightarrow P$ is a mixed monotone operator and $T(h, h)=A h+B(h, h) \in P_{h}$. Moreover, for any $x, y \in P$ and $t \in(0,1)$, we have

$$
\begin{aligned}
T\left(t x, t^{-1} y\right) & =A(t x)+B\left(t x, t^{-1} y\right) \geq \varphi_{1}(t) A x+\varphi_{2}(t) B(x, y) \\
& \geq \varphi(t)[A x+B(x, y)]=\varphi(t) T(x, y) .
\end{aligned}
$$

Hence, all the conditions of Theorem 1.2 are satisfied. An application of Theorem $1.2 \mathrm{im}-$ plies that: there are $u_{0}, v_{0} \in P_{h}$ and $r \in(0,1)$ such that $r v_{0} \leq u_{0}<v_{0}, u_{0} \leq T\left(u_{0}, v_{0}\right) \leq$ $T\left(v_{0}, u_{0}\right) \leq v_{0}$; operator equation $T(x, x)=x$ has a unique solution $x^{*} \in P_{h}$; for any initial values $x_{0}, y_{0} \in P_{h}$, constructing successively the sequences

$$
x_{n}=T\left(x_{n-1}, y_{n-1}\right), \quad y_{n}=T\left(y_{n-1}, x_{n-1}\right), \quad n=1,2, \ldots,
$$

we have $\left\|x_{n}-x^{*}\right\| \rightarrow 0$ and $\left\|y_{n}-x^{*}\right\| \rightarrow 0$ as $n \rightarrow \infty$. That is:

(i) there exist $u_{0}, v_{0} \in P_{h}$ and $r \in(0,1)$ such that

$$
r v_{0} \leq u_{0}<v_{0}, \quad u_{0} \leq A u_{0}+B\left(u_{0}, v_{0}\right) \leq A v_{0}+B\left(v_{0}, u_{0}\right) \leq v_{0}
$$

(ii) the operator equation (2.6) has a unique solution $x^{*}$ in $P_{h}$;

(iii) for any initial values $x_{0}, y_{0} \in P_{h}$, constructing successively the sequences

$$
x_{n}=A x_{n-1}+B\left(x_{n-1}, y_{n-1}\right), \quad y_{n}=A y_{n-1}+B\left(y_{n-1}, x_{n-1}\right), \quad n=1,2, \ldots,
$$

we have $x_{n} \rightarrow x^{*}, y_{n} \rightarrow x^{*}$ as $n \rightarrow \infty$.

Corollary 2.8 Let $P$ be a normal cone, $A: P_{h} \rightarrow P_{h}$ be an increasing operator and $B$ : $P_{h} \times P_{h} \rightarrow P_{h}$ be a mixed monotone operator. Assume that:

$\left(\mathrm{H}_{24}\right)$ for any $x \in P_{h}, t \in(0,1)$, there exists $\varphi_{1}(t) \in(t, 1)$ such that

$$
A(t x) \geq \varphi_{1}(t) A x ;
$$

$\left(\mathrm{H}_{25}\right)$ for any $x, y \in P_{h}, t \in(0,1)$, there exists $\varphi_{2}(t) \in(t, 1)$ such that

$$
B\left(t x, t^{-1} y\right) \geq \varphi_{2}(t) B(x, y)
$$


Then:

(i) there exist $u_{0}, v_{0} \in P_{h}$ and $r \in(0,1)$ such that

$$
r v_{0} \leq u_{0}<v_{0}, \quad u_{0} \leq A u_{0}+B\left(u_{0}, v_{0}\right) \leq A v_{0}+B\left(v_{0}, u_{0}\right) \leq v_{0}
$$

(ii) the operator equation (2.6) has a unique solution $x^{*}$ in $P_{h}$;

(iii) for any initial values $x_{0}, y_{0} \in P_{h}$, constructing successively the sequences

$$
x_{n}=A x_{n-1}+B\left(x_{n-1}, y_{n-1}\right), \quad y_{n}=A y_{n-1}+B\left(y_{n-1}, x_{n-1}\right), \quad n=1,2, \ldots,
$$

we have $x_{n} \rightarrow x^{*}, y_{n} \rightarrow x^{*}$ as $n \rightarrow \infty$.

Corollary 2.9 Let $\alpha \in(0,1)$ and $P$ be a normal cone. Let $A: P \rightarrow P$ be an increasing operator which satisfies $\left(\mathrm{H}_{21}\right), B_{1}: P \rightarrow P$ be an increasing sub-homogeneous operator and $B_{2}: P \times P \rightarrow P$ be a mixed monotone operator which satisfies

$$
B_{2}\left(t x, t^{-1} y\right) \geq t^{\alpha} B_{2}(x, y), \quad t \in(0,1), x, y \in P .
$$

Assume that:

$\left(\mathrm{H}_{26}\right)$ there exist $h_{0}, h_{1} \in P_{h}$ such that

$$
A h_{0} \in P_{h}, \quad B_{1} h_{1}+B_{2}\left(h_{1}, h_{1}\right) \in P_{h} ;
$$

$\left(\mathrm{H}_{27}\right)$ there exists a constant $\delta_{0}>0$ such that

$$
B_{2}(x, y) \geq \delta_{0} B_{1} x, \quad x, y \in P .
$$

Then:

(i) there exist $u_{0}, v_{0} \in P_{h}$ and $r \in(0,1)$ such that

$$
\begin{aligned}
& r v_{0} \leq u_{0}<v_{0}, \\
& u_{0} \leq A u_{0}+B_{1} u_{0}+B_{2}\left(u_{0}, v_{0}\right) \leq A v_{0}+B_{1} v_{0}+B_{2}\left(v_{0}, u_{0}\right) \leq v_{0}
\end{aligned}
$$

(ii) the following operator equation:

$$
A x+B_{1} x+B_{2}(x, x)=x
$$

has a unique solution $x^{*}$ in $P_{h}$;

(iii) for any initial values $x_{0}, y_{0} \in P_{h}$, constructing successively the sequences

$$
\begin{aligned}
& x_{n}=A x_{n-1}+B_{1} x_{n-1}+B_{2}\left(x_{n-1}, y_{n-1}\right), \\
& y_{n}=A y_{n-1}+B_{1} y_{n-1}+B_{2}\left(y_{n-1}, x_{n-1}\right), \quad n=1,2, \ldots,
\end{aligned}
$$

we have $x_{n} \rightarrow x^{*}, y_{n} \rightarrow x^{*}$ as $n \rightarrow \infty$. 
Proof Define an operator $B=B_{1}+B_{2}$ by $B(x, y)=B_{1} x+B_{2}(x, y)$. Then $B: P \times P \rightarrow P$ is a mixed monotone operator and $B\left(h_{1}, h_{1}\right)=B_{1} h_{1}+B_{2}\left(h_{1}, h_{1}\right) \in P_{h}$. Since $h_{0}, h_{1} \in P_{h}$, there exist $t_{0}, t_{1} \in(0,1)$ such that

$$
t_{0} h \leq h_{0} \leq \frac{1}{t_{0}} h, \quad t_{1} h \leq h_{1} \leq \frac{1}{t_{1}} h .
$$

Then $h_{1} \leq \frac{1}{t_{1}} h \leq \frac{1}{t_{0} t_{1}} h_{0}, h_{1} \geq t_{1} h \geq t_{0} t_{1} h_{0}$, and thus

$$
A h_{1} \geq A\left(t_{0} t_{1} h_{0}\right) \geq \varphi_{1}\left(t_{0} t_{1}\right) A h_{0}, \quad A h_{1} \leq A\left(\frac{1}{t_{0} t_{1}} h_{0}\right) \leq \frac{1}{\varphi_{1}\left(t_{0} t_{1}\right)} A h_{0} .
$$

Note that $\varphi_{1}\left(t_{0} t_{1}\right), \frac{1}{\varphi_{1}\left(t_{0} t_{1}\right)}>0$ and $A h_{0} \in P_{h}$, we can get $A h_{1} \in P_{h}$. Hence, $A h_{1}+B\left(h_{1}, h_{1}\right) \in P_{h}$.

Note that $\left(\mathrm{H}_{27}\right)$ and from the proof of Theorem 1.4, there exists $\beta_{0}(t) \in(\alpha, 1)$ with respect to $t$ such that

$$
B\left(t x, t^{-1} y\right) \geq t^{\beta_{0}(t)} B(x, y), \quad \forall t \in(0,1), x, y \in P .
$$

Let $\varphi_{2}(t)=t^{\beta_{0}(t)}, t \in(0,1)$. Then $\varphi_{2}(t) \in(t, 1)$ and

$$
B\left(t x, t^{-1} y\right) \geq \varphi_{2}(t) B(x, y), \quad \forall t \in(0,1), x, y \in P .
$$

Therefore, the operators $A, B$ satisfy all the conditions of Theorem 2.7. So we easily obtain the following conclusions:

(i) there exist $u_{0}, v_{0} \in P_{h}$ and $r \in(0,1)$ such that

$$
\begin{aligned}
& r v_{0} \leq u_{0}<v_{0}, \\
& u_{0} \leq A u_{0}+B_{1} u_{0}+B_{2}\left(u_{0}, v_{0}\right) \leq A v_{0}+B_{1} v_{0}+B_{2}\left(v_{0}, u_{0}\right) \leq v_{0} ;
\end{aligned}
$$

(ii) the operator equation (2.12) has a unique solution $x^{*}$ in $P_{h}$;

(iii) for any initial values $x_{0}, y_{0} \in P_{h}$, constructing successively the sequences

$$
\begin{aligned}
& x_{n}=A x_{n-1}+B_{1} x_{n-1}+B_{2}\left(x_{n-1}, y_{n-1}\right), \\
& y_{n}=A y_{n-1}+B_{1} y_{n-1}+B_{2}\left(y_{n-1}, x_{n-1}\right), \quad n=1,2, \ldots,
\end{aligned}
$$

we have $x_{n} \rightarrow x^{*}, y_{n} \rightarrow x^{*}$ as $n \rightarrow \infty$.

Corollary 2.10 Let $\alpha \in(0,1)$ and $P$ be a normal cone. Let $A: P \rightarrow P$ be an increasing operator which satisfies $\left(\mathrm{H}_{21}\right), B_{1}: P \rightarrow P$ be an increasing $\alpha$-concave operator and $B_{2}$ : $P \times P \rightarrow P$ be a mixed monotone operator which satisfies

$$
B_{2}\left(t x, t^{-1} y\right) \geq t B_{2}(x, y), \quad t \in(0,1), x, y \in P .
$$

Assume that $\left(\mathrm{H}_{26}\right)$ holds and

$\left(\mathrm{H}_{28}\right)$ there exists a constant $\delta_{0}>0$ such that $B_{2}(x, y) \leq \delta_{0} B_{1} x, \forall x, y \in P$. 
Then:

(i) there exist $u_{0}, v_{0} \in P_{h}$ and $r \in(0,1)$ such that

$$
r v_{0} \leq u_{0}<v_{0}, \quad u_{0} \leq A u_{0}+B_{1} u_{0}+B_{2}\left(u_{0}, v_{0}\right) \leq A v_{0}+B_{1} v_{0}+B_{2}\left(v_{0}, u_{0}\right) \leq v_{0}
$$

(ii) the operator equation (2.12) has a unique solution $x^{*}$ in $P_{h}$;

(iii) for any initial values $x_{0}, y_{0} \in P_{h}$, constructing successively the sequences

$$
\begin{aligned}
& x_{n}=A x_{n-1}+B_{1} x_{n-1}+B_{2}\left(x_{n-1}, y_{n-1}\right), \\
& y_{n}=A y_{n-1}+B_{1} y_{n-1}+B_{2}\left(y_{n-1}, x_{n-1}\right), \quad n=1,2, \ldots,
\end{aligned}
$$

we have $x_{n} \rightarrow x^{*}, y_{n} \rightarrow x^{*}$ as $n \rightarrow \infty$.

Proof Consider the same operator $B$ defined by the proof of Corollary 2.9, we have $B$ : $P \times P \rightarrow P$ is a mixed monotone operator and $B\left(h_{1}, h_{1}\right) \in P_{h}$. From Definition 1.1, we have $A(t x) \leq \frac{1}{t^{\alpha}} A x, t \in(0,1), x \in P$. Since $h_{0}, h_{1} \in P_{h}$, there exist $t_{0}, t_{1} \in(0,1)$ such that

$$
t_{0} h \leq h_{0} \leq \frac{1}{t_{0}} h, \quad t_{1} h \leq h_{1} \leq \frac{1}{t_{1}} h .
$$

Then $t_{0} t_{1} h_{0} \leq h_{1} \leq \frac{1}{t_{0} t_{1}} h_{0}$, and thus

$$
A h_{1} \geq A\left(t_{0} t_{1} h_{0}\right) \geq\left(t_{0} t_{1}\right)^{\alpha} A h_{0}, \quad A h_{1} \leq A\left(\frac{1}{t_{0} t_{1}} h_{0}\right) \leq \frac{1}{\left(t_{0} t_{1}\right)^{\alpha}} A h_{0} .
$$

Note that $\left(t_{0} t_{1}\right)^{\alpha}, \frac{1}{\left(t_{0} t_{1}\right)^{\alpha}}>0$ and $A h_{0} \in P_{h}$, we can get $A h_{1} \in P_{h}$. Hence, $A h_{1}+B\left(h_{1}, h_{1}\right) \in P_{h}$.

Note that $\left(\mathrm{H}_{28}\right)$ and from the proof of Theorem 1.5, we know that there exists $\beta_{0}(t) \in$ $(\alpha, 1)$ with respect to $t$ such that

$$
B\left(t x, t^{-1} y\right) \geq t^{\beta_{0}(t)} B(x, y), \quad \forall t \in(0,1), x, y \in P .
$$

Let $\varphi_{2}(t)=t^{\beta_{0}(t)}, t \in(0,1)$. Then $\varphi_{2}(t) \in(t, 1)$ and $B\left(t x, t^{-1} y\right) \geq \varphi_{2}(t) B(x, y), x, y \in P$.

Therefore, the operators $A, B$ satisfy all the conditions of Theorem 2.7. So we easily obtain the following conclusions:

(i) there exist $u_{0}, v_{0} \in P_{h}$ and $r \in(0,1)$ such that

$$
\begin{aligned}
& r v_{0} \leq u_{0}<v_{0}, \\
& u_{0} \leq A u_{0}+B_{1} u_{0}+B_{2}\left(u_{0}, v_{0}\right) \leq A v_{0}+B_{1} v_{0}+B_{2}\left(v_{0}, u_{0}\right) \leq v_{0} ;
\end{aligned}
$$

(ii) the operator equation (2.12) has a unique solution $x^{*}$ in $P_{h}$;

(iii) for any initial values $x_{0}, y_{0} \in P_{h}$, constructing successively the sequences

$$
\begin{aligned}
& x_{n}=A x_{n-1}+B_{1} x_{n-1}+B_{2}\left(x_{n-1}, y_{n-1}\right), \\
& y_{n}=A y_{n-1}+B_{1} y_{n-1}+B_{2}\left(y_{n-1}, x_{n-1}\right), \quad n=1,2, \ldots,
\end{aligned}
$$

we have $x_{n} \rightarrow x^{*}, y_{n} \rightarrow x^{*}$ as $n \rightarrow \infty$.

Similar to Corollary 2.5, we have the following result. 
Corollary 2.11 Assume that all the conditions of Theorem 2.7 hold. Let $x_{\lambda}(\lambda>0)$ denote the unique solution of operator equation (2.7). Then we have the following conclusions:

(i) if $\varphi_{i}(t)>t^{\frac{1}{2}}(i=1,2)$ for $t \in(0,1)$, then $x_{\lambda}$ is strictly decreasing in $\lambda$, that is, $0<\lambda_{1}<\lambda_{2}$ implies $x_{\lambda_{1}}>x_{\lambda_{2}}$

(ii) if there exists $\beta \in(0,1)$ such that $\varphi_{i}(t) \geq t^{\beta}(i=1,2)$ for $t \in(0,1)$, then $x_{\lambda}$ is continuous in $\lambda$, that is, $\lambda \rightarrow \lambda_{0}\left(\lambda_{0}>0\right)$ implies $\left\|x_{\lambda}-x_{\lambda_{0}}\right\| \rightarrow 0$;

(iii) if there exists $\beta \in\left(0, \frac{1}{2}\right)$ such that $\varphi_{i}(t) \geq t^{\beta}(i=1,2)$ for $t \in(0,1)$, then $\lim _{\lambda \rightarrow \infty}\left\|x_{\lambda}\right\|=0, \lim _{\lambda \rightarrow 0^{+}}\left\|x_{\lambda}\right\|=\infty$.

\subsection{The sum of decreasing operators and mixed monotone operators}

In the following we also consider the operator equations (2.6) and (2.7).

Theorem 2.12 Let $P$ be a normal cone, $A: P \rightarrow P$ be a decreasing operator and $B: P \times P \rightarrow$ $P$ be a mixed monotone operator. Assume that $\left(\mathrm{H}_{22}\right)$ and $\left(\mathrm{H}_{23}\right)$ hold and

$\left(\mathrm{H}_{31}\right)$ for any $x \in P$ and $t \in(0,1)$, there exists $\varphi_{1}(t) \in(t, 1)$ such that

$$
A(t x) \leq \frac{1}{\varphi_{1}(t)} A x .
$$

Then:

(i) there exist $u_{0}, v_{0} \in P_{h}$ and $r \in(0,1)$ such that

$$
r v_{0} \leq u_{0}<v_{0}, \quad u_{0} \leq A v_{0}+B\left(u_{0}, v_{0}\right) \leq A u_{0}+B\left(v_{0}, u_{0}\right) \leq v_{0} ;
$$

(ii) the operator equation (2.6) has a unique solution $x^{*}$ in $P_{h}$;

(iii) for any initial values $x_{0}, y_{0} \in P_{h}$, constructing successively the sequences

$$
x_{n}=A y_{n-1}+B\left(x_{n-1}, y_{n-1}\right), \quad y_{n}=A x_{n-1}+B\left(y_{n-1}, x_{n-1}\right), \quad n=1,2, \ldots,
$$

we have $x_{n} \rightarrow x^{*}, y_{n} \rightarrow x^{*}$ as $n \rightarrow \infty$.

Proof From (2.14), we have

$$
A\left(\frac{1}{t} x\right) \geq \varphi_{1}(t) A x, \quad t \in(0,1), x \in P .
$$

Since $A h_{0}+B\left(h_{0}, h_{0}\right) \in P_{h}$, there exist constants $\lambda_{1}, \lambda_{2}>0$ such that

$$
\lambda_{1} h \leq A h_{0}+B\left(h_{0}, h_{0}\right) \leq \lambda_{2} h
$$

Also from $h_{0} \in P_{h}$, there exists a small constant $t_{0} \in(0,1)$ such that

$$
t_{0} h \leq h_{0} \leq \frac{1}{t_{0}} h .
$$

Let $\varphi(t)=\min \left\{\varphi_{1}(t), \varphi_{2}(t)\right\}$. Then $\varphi(t) \in(t, 1)$ for $t \in(0,1)$. From $\left(\mathrm{H}_{22}\right)$ and $(2.14),(2.15)$,

$$
\begin{aligned}
A h+B(h, h) & \geq A\left(\frac{1}{t_{0}} h_{0}\right)+B\left(t_{0} h_{0}, t_{0}^{-1} h_{0}\right) \geq \varphi_{1}\left(t_{0}\right) A h_{0}+\varphi_{2}\left(t_{0}\right) B\left(h_{0}, h_{0}\right) \\
& \geq \varphi\left(t_{0}\right)\left[A h_{0}+B\left(h_{0}, h_{0}\right)\right] \geq \lambda_{1} \varphi\left(t_{0}\right) h,
\end{aligned}
$$




$$
\begin{aligned}
A h+B(h, h) & \leq A\left(t_{0} h_{0}\right)+B\left(t_{0}^{-1} h_{0}, t_{0} h_{0}\right) \leq \frac{1}{\varphi_{1}\left(t_{0}\right)} A h_{0}+\frac{1}{\varphi_{2}\left(t_{0}\right)} B\left(h_{0}, h_{0}\right) \\
& \leq \frac{1}{\varphi\left(t_{0}\right)}\left[A h_{0}+B\left(h_{0}, h_{0}\right)\right] \leq \frac{\lambda_{2}}{\varphi\left(t_{0}\right)} h .
\end{aligned}
$$

Note that $\lambda_{1} \varphi\left(t_{0}\right), \frac{\lambda_{2}}{\varphi\left(t_{0}\right)}>0$, we can get $A h+B(h, h) \in P_{h}$.

Next, we define an operator $T=A+B$ by $T(x, y)=A y+B(x, y)$. Then $T: P \times P \rightarrow P$ is a mixed monotone operator and $T(h, h)=A h+B(h, h) \in P_{h}$.

Moreover, for any $x, y \in P$ and $t \in(0,1)$, we have

$$
\begin{aligned}
T\left(t x, t^{-1} y\right) & =A\left(t^{-1} y\right)+B\left(t x, t^{-1} y\right) \geq \varphi_{1}(t) A y+\varphi_{2}(t) B(x, y) \\
& \geq \varphi(t)[A y+B(x, y)]=\varphi(t) T(x, y) .
\end{aligned}
$$

Hence, all the conditions of Theorem 1.2 are satisfied. Application of Theorem 1.2 implies that: there are $u_{0}, v_{0} \in P_{h}$ and $r \in(0,1)$ such that $r v_{0} \leq u_{0}<v_{0}, u_{0} \leq T\left(u_{0}, v_{0}\right) \leq T\left(v_{0}, u_{0}\right) \leq$ $v_{0}$; operator equation $T(x, x)=x$ has a unique solution $x^{*} \in P_{h}$; for any initial values $x_{0}, y_{0} \in$ $P_{h}$, constructing successively the sequences

$$
x_{n}=T\left(x_{n-1}, y_{n-1}\right), \quad y_{n}=T\left(y_{n-1}, x_{n-1}\right), \quad n=1,2, \ldots,
$$

we have $\left\|x_{n}-x^{*}\right\| \rightarrow 0$ and $\left\|y_{n}-x^{*}\right\| \rightarrow 0$ as $n \rightarrow \infty$. That is,

(i) there exist $u_{0}, v_{0} \in P_{h}$ and $r \in(0,1)$ such that

$$
r v_{0} \leq u_{0}<v_{0}, \quad u_{0} \leq A v_{0}+B\left(u_{0}, v_{0}\right) \leq A u_{0}+B\left(v_{0}, u_{0}\right) \leq v_{0}
$$

(ii) the operator equation (2.6) has a unique solution $x^{*}$ in $P_{h}$;

(iii) for any initial values $x_{0}, y_{0} \in P_{h}$, constructing successively the sequences

$$
x_{n}=A y_{n-1}+B\left(x_{n-1}, y_{n-1}\right), \quad y_{n}=A x_{n-1}+B\left(y_{n-1}, x_{n-1}\right), \quad n=1,2, \ldots,
$$

we have $x_{n} \rightarrow x^{*}, y_{n} \rightarrow x^{*}$ as $n \rightarrow \infty$.

Corollary 2.13 Let $P$ be a normal cone, $A: P_{h} \rightarrow P_{h}$ be a decreasing operator and $B:$ $P_{h} \times P_{h} \rightarrow P_{h}$ be a mixed monotone operator. Assume that:

$\left(\mathrm{H}_{32}\right)$ for any $x \in P_{h}$ and $t \in(0,1)$, there exists $\varphi_{1}(t) \in(t, 1)$ such that

$$
A(t x) \leq \frac{1}{\varphi_{1}(t)} A x
$$

$\left(\mathrm{H}_{33}\right)$ for any $x, y \in P_{h}, t \in(0,1)$, there exists $\varphi_{2}(t) \in(t, 1)$ such that

$$
B\left(t x, t^{-1} y\right) \geq \varphi_{2}(t) B(x, y) .
$$

Then:

(i) there exist $u_{0}, v_{0} \in P_{h}$ and $r \in(0,1)$ such that

$$
r v_{0} \leq u_{0}<v_{0}, \quad u_{0} \leq A v_{0}+B\left(u_{0}, v_{0}\right) \leq A u_{0}+B\left(v_{0}, u_{0}\right) \leq v_{0} ;
$$


(ii) the operator equation (2.6) has a unique solution $x^{*}$ in $P_{h}$;

(iii) for any initial values $x_{0}, y_{0} \in P_{h}$, constructing successively the sequences

$$
x_{n}=A y_{n-1}+B\left(x_{n-1}, y_{n-1}\right), \quad y_{n}=A x_{n-1}+B\left(y_{n-1}, x_{n-1}\right), \quad n=1,2, \ldots,
$$

we have $x_{n} \rightarrow x^{*}, y_{n} \rightarrow x^{*}$ as $n \rightarrow \infty$.

Corollary 2.14 Assume that all the conditions of Theorem 2.12 hold. Let $x_{\lambda}(\lambda>0)$ denote the unique solution of operator equation (2.7). Then we have the following conclusions:

(i) if $\varphi_{i}(t)>t^{\frac{1}{2}}(i=1,2)$ for $t \in(0,1)$, then $x_{\lambda}$ is strictly decreasing in $\lambda$, that is, $0<\lambda_{1}<\lambda_{2}$ implies $x_{\lambda_{1}}>x_{\lambda_{2}}$;

(ii) if there exists $\beta \in(0,1)$ such that $\varphi_{i}(t) \geq t^{\beta}(i=1,2)$ for $t \in(0,1)$, then $x_{\lambda}$ is continuous in $\lambda$, that is, $\lambda \rightarrow \lambda_{0}\left(\lambda_{0}>0\right)$ implies $\left\|x_{\lambda}-x_{\lambda_{0}}\right\| \rightarrow 0$;

(iii) if there exists $\beta \in\left(0, \frac{1}{2}\right)$ such that $\varphi_{i}(t) \geq t^{\beta}(i=1,2)$ for $t \in(0,1)$, then $\lim _{\lambda \rightarrow \infty}\left\|x_{\lambda}\right\|=0, \lim _{\lambda \rightarrow 0^{+}}\left\|x_{\lambda}\right\|=\infty$.

\subsection{The sum of increasing operators, decreasing operators, and mixed monotone operators}

From the above results, we can easily obtain the following results on operator equations:

$$
\begin{aligned}
& A x+B_{1} x+B_{2}(x, x)=x, \\
& A_{1} x+A_{2} x+B_{1} x+B_{2}(x, x)=x .
\end{aligned}
$$

By Theorem 2.12 and Corollary 2.9, Corollary 2.10, we have the following conclusions.

Theorem 2.15 Let $\alpha \in(0,1)$ and $P$ be a normal cone. Let $A: P \rightarrow P$ be a decreasing operator which satisfies $\left(\mathrm{H}_{31}\right)$, operators $B_{1}, B_{2}$ be the same as for Corollary 2.9. Assume that $\left(\mathrm{H}_{26}\right),\left(\mathrm{H}_{27}\right)$ hold. Then:

(i) there exist $u_{0}, v_{0} \in P_{h}$ and $r \in(0,1)$ such that

$$
\begin{aligned}
& r v_{0} \leq u_{0}<v_{0}, \\
& u_{0} \leq A v_{0}+B_{1} u_{0}+B_{2}\left(u_{0}, v_{0}\right) \leq A u_{0}+B_{1} v_{0}+B_{2}\left(v_{0}, u_{0}\right) \leq v_{0} ;
\end{aligned}
$$

(ii) the operator equation (2.16) has a unique solution $x^{*}$ in $P_{h}$;

(iii) for any initial values $x_{0}, y_{0} \in P_{h}$, constructing successively the sequences

$$
\begin{aligned}
& x_{n}=A y_{n-1}+B_{1} x_{n-1}+B_{2}\left(x_{n-1}, y_{n-1}\right), \\
& y_{n}=A x_{n-1}+B_{1} y_{n-1}+B_{2}\left(y_{n-1}, x_{n-1}\right), \quad n=1,2, \ldots,
\end{aligned}
$$

we have $x_{n} \rightarrow x^{*}, y_{n} \rightarrow x^{*}$ as $n \rightarrow \infty$.

Theorem 2.16 Let $\alpha \in(0,1)$ and $P$ be a normal cone. Let $A: P \rightarrow P$ be a decreasing operator which satisfies $\left(\mathrm{H}_{31}\right)$, operators $B_{1}, B_{2}$ be the same as for Corollary 2.10. Assume that $\left(\mathrm{H}_{26}\right),\left(\mathrm{H}_{28}\right)$ hold. Then: 
(i) there exist $u_{0}, v_{0} \in P_{h}$ and $r \in(0,1)$ such that

$$
\begin{aligned}
& r v_{0} \leq u_{0}<v_{0} \\
& u_{0} \leq A v_{0}+B_{1} u_{0}+B_{2}\left(u_{0}, v_{0}\right) \leq A u_{0}+B_{1} v_{0}+B_{2}\left(v_{0}, u_{0}\right) \leq v_{0}
\end{aligned}
$$

(ii) the operator equation (2.16) has a unique solution $x^{*}$ in $P_{h}$;

(iii) for any initial values $x_{0}, y_{0} \in P_{h}$, constructing successively the sequences

$$
\begin{aligned}
& x_{n}=A y_{n-1}+B_{1} x_{n-1}+B_{2}\left(x_{n-1}, y_{n-1}\right), \\
& y_{n}=A x_{n-1}+B_{1} y_{n-1}+B_{2}\left(y_{n-1}, x_{n-1}\right), \quad n=1,2, \ldots,
\end{aligned}
$$

we have $x_{n} \rightarrow x^{*}, y_{n} \rightarrow x^{*}$ as $n \rightarrow \infty$.

From Corollary 2.9, Corollary 2.10, and Corollary 2.4, we can easily obtain the following results.

Theorem 2.17 Let $\alpha_{1}, \alpha_{2} \in(0,1)$ and P be a normal cone, operators $A_{1}, A_{2}$ satisfy the conditions of Corollary 2.4, where $A_{1}$ is $\alpha_{1}$-concave, operators $B_{1}, B_{2}$ satisfy the conditions of Corollary 2.9, where $B_{2}$ satisfies (2.11) with $\alpha$ replaced by $\alpha_{2}$. Then:

(i) there exist $u_{0}, v_{0} \in P_{h}$ and $r \in(0,1)$ such that

$$
\begin{aligned}
& r v_{0} \leq u_{0}<v_{0} \\
& u_{0} \leq A_{1} u_{0}+A_{2} u_{0}+B_{1} u_{0}+B_{2}\left(u_{0}, v_{0}\right) \leq A_{1} v_{0}+A_{2} v_{0}+B_{1} v_{0}+B_{2}\left(v_{0}, u_{0}\right) \leq v_{0}
\end{aligned}
$$

(ii) the operator equation (2.17) has a unique solution $x^{*}$ in $P_{h}$;

(iii) for any initial values $x_{0}, y_{0} \in P_{h}$, constructing successively the sequences

$$
\begin{aligned}
& x_{n}=A_{1} x_{n-1}+A_{2} x_{n-1}+B_{1} x_{n-1}+B_{2}\left(x_{n-1}, y_{n-1}\right), \\
& y_{n}=A_{1} y_{n-1}+A_{2} y_{n-1}+B_{1} y_{n-1}+B_{2}\left(y_{n-1}, x_{n-1}\right),
\end{aligned}
$$

where $n=1,2, \ldots$, we have $x_{n} \rightarrow x^{*}, y_{n} \rightarrow x^{*}$ as $n \rightarrow \infty$.

Theorem 2.18 Let $\alpha_{1}, \alpha_{2} \in(0,1)$ and $P$ be a normal cone, operator $A: P \rightarrow P$ is $\alpha_{1}$-concave, operators $B_{1}, B_{2}$ satisfy the conditions of Corollary 2.10, where $B_{1}$ is $\alpha_{2}$-concave. Then:

(i) there exist $u_{0}, v_{0} \in P_{h}$ and $r \in(0,1)$ such that

$$
\begin{aligned}
& r v_{0} \leq u_{0}<v_{0} \\
& u_{0} \leq A_{1} u_{0}+A_{2} u_{0}+B_{1} u_{0}+B_{2}\left(u_{0}, v_{0}\right) \leq A_{1} v_{0}+A_{2} v_{0}+B_{1} v_{0}+B_{2}\left(v_{0}, u_{0}\right) \leq v_{0}
\end{aligned}
$$

(ii) the operator equation (2.17) has a unique solution $x^{*}$ in $P_{h}$;

(iii) for any initial values $x_{0}, y_{0} \in P_{h}$, constructing successively the sequences

$$
\begin{aligned}
& x_{n}=A_{1} x_{n-1}+A_{2} x_{n-1}+B_{1} x_{n-1}+B_{2}\left(x_{n-1}, y_{n-1}\right), \\
& y_{n}=A_{1} y_{n-1}+A_{2} y_{n-1}+B_{1} y_{n-1}+B_{2}\left(y_{n-1}, x_{n-1}\right),
\end{aligned}
$$

where $n=1,2, \ldots$, we have $x_{n} \rightarrow x^{*}, y_{n} \rightarrow x^{*}$ as $n \rightarrow \infty$. 


\section{Some applications}

In this section, we will apply the main results to study nonlinear problems which include nonlinear integral equations and nonlinear elliptic boundary value problems for the Lane-Emden-Fowler equations. And then we will obtain new results on the existence and uniqueness of positive solutions for these problems, which are not consequences of the corresponding fixed point theorems in the literature.

\subsection{Applications to nonlinear integral equations}

A standard approach, in studying the existence of positive solutions of boundary value problems (BVPs for short) for ordinary differential equations, is to rewrite the problem as an equivalent positive-solution problem for a Hammerstein integral equation of the form

$$
u(t)=\lambda \int_{a}^{b} G(t, s) f(s, u(s)) d s
$$

in the space $E=C[a, b]$, where the nonlinearity $f$ and the kernel $G$ (the Green function of the problem) are both nonnegative, $\lambda>0$ is a parameter. One seeks fixed points of a Hammerstein integral operator in a suitable cone of positive functions.

Set $P=\{u \in C[a, b] \mid u(t) \geq 0, t \in[a, b]\}$, the standard cone. It is easy to see that $P$ is a normal cone of which the normality constant is 1 . Then $P_{h}=\left\{x \in P \mid\right.$ there are $\lambda_{2}(x) \geq \lambda_{1}(x)>$ 0 such that $\left.\lambda_{1}(x) h(t) \leq x(t) \leq \lambda_{2}(x) h(t), t \in[a, b]\right\}$. Assume that $G(t, s):[a, b] \times[a, b] \rightarrow$ $[0,+\infty)$ is continuous with $G(t, s) \not \equiv 0$ and there exist $h, m, n \in C([a, b],[0,+\infty))$ with $h(t), m(t), n(t) \not \equiv 0$, such that

$$
m(s) h(t) \leq G(t, s) \leq n(s) h(t) \quad \text { for all } t, s \in[a, b] .
$$

Theorem 3.1 Assume that $f(t, x)=f_{1}(t, x)+f_{2}(t, x) \not \equiv 0$ and

$\left(\mathrm{H}_{31}\right) f_{i}:[a, b] \times[0,+\infty) \rightarrow[0,+\infty)$ is continuous $(i=1,2), f_{1}(t, x)$ is increasing in $x \in$ $[0,+\infty)$ for fixed $t \in[a, b]$ and $f_{2}(t, x)$ is decreasing in $x \in[0,+\infty)$ for fixed $t \in[a, b]$;

$\left(\mathrm{H}_{32}\right)$ for $\eta \in(0,1)$, there exist $\varphi_{i}(\eta) \in(\eta, 1)(i=1,2)$ such that

$$
f_{1}(t, \eta x) \geq \varphi_{1}(\eta) f_{1}(t, x), \quad f_{2}(t, \eta x) \leq \frac{1}{\varphi_{2}(\eta)} f_{2}(t, x), \quad \forall t \in[a, b], x \in[0,+\infty) .
$$

Then, for any given $\lambda>0$, the integral equation (3.1) has a unique positive solution $u_{\lambda}^{*}$ in $P_{h}$. Moreover, for any initial values $x_{0}, y_{0} \in P_{h}$, constructing successively the sequences:

$$
\begin{aligned}
& x_{n}=\lambda \int_{a}^{b} G(t, s)\left[f_{1}\left(s, x_{n-1}(s)\right)+f_{2}\left(s, y_{n-1}(s)\right)\right] d s, \\
& y_{n}=\lambda \int_{a}^{b} G(t, s)\left[f_{1}\left(s, y_{n-1}(s)\right)+f_{2}\left(s, x_{n-1}(s)\right)\right] d s, \quad n=1,2, \ldots
\end{aligned}
$$

we have $x_{n} \rightarrow u_{\lambda}^{*}, y_{n} \rightarrow u_{\lambda}^{*}$ as $n \rightarrow+\infty$. Further, (i) if $\varphi_{i}(t)>t^{\frac{1}{2}}(i=1,2)$ for $t \in(0,1)$, then $u_{\lambda}^{*}$ is strictly increasing in $\lambda$, that is, $0<\lambda_{1}<\lambda_{2}$ implies $u_{\lambda_{1}}^{*}<u_{\lambda_{2}}^{*}$; (ii) if there exists $\beta \in(0,1)$ such that $\varphi_{i}(t) \geq t^{\beta}(i=1,2)$ for $t \in(0,1)$, then $u_{\lambda}^{*}$ is continuous in $\lambda$, that is, $\lambda \rightarrow \lambda_{0}\left(\lambda_{0}>\right.$ $0)$ implies $\left\|u_{\lambda}^{*}-u_{\lambda_{0}}^{*}\right\| \rightarrow 0$; (iii) if there exists $\beta \in\left(0, \frac{1}{2}\right)$ such that $\varphi_{i}(t) \geq t^{\beta}(i=1,2)$ for $t \in(0,1)$, then $\lim _{\lambda \rightarrow 0^{+}}\left\|u_{\lambda}^{*}\right\|=0, \lim _{\lambda \rightarrow+\infty}\left\|u_{\lambda}^{*}\right\|=+\infty$. 
Proof Define two operators $A: P \rightarrow E$ and $B: P \rightarrow E$ by

$$
A u(t)=\int_{a}^{b} G(t, s) f_{1}(s, u(s)) d s, \quad B u(t)=\int_{a}^{b} G(t, s) f_{2}(s, u(s)) d s .
$$

It is easy to see that $u$ is the solution of (3.1) if and only if $u=\lambda(A u+B u)$. From $\left(\mathrm{H}_{31}\right)$, we know that $A: P \rightarrow P$ is increasing and $B: P \rightarrow P$ is decreasing. Further, from $\left(\mathrm{H}_{32}\right)$, we can prove that $A, B$ satisfy $\left(\mathrm{H}_{11}\right)$. Next we prove that $A h+B h \in P_{h}$. Set $h_{\max }=\max _{t \in[a, b]} h(t)$, $h_{\min }=\min _{t \in[a, b]} h(t)$. Then $h_{\max } \geq h_{\min }>0$.

For any $t \in[a, b]$, from $\left(\mathrm{H}_{31}\right)$ and (3.2), we have

$$
\begin{aligned}
A h(t)+B h(t) & =\int_{a}^{b} G(t, s)\left[f_{1}(s, h(s))+f_{2}(s, h(s))\right] d s \\
& \geq \int_{a}^{b} h(t) m(s)\left[f_{1}\left(s, h_{\text {min }}\right)+f_{2}\left(s, h_{\text {max }}\right)\right] d s \\
& =h(t) \int_{a}^{b} m(s)\left[f_{1}\left(s, h_{\text {min }}\right)+f_{2}\left(s, h_{\text {max }}\right)\right] d s, \\
A h(t)+B h(t) & \leq \int_{a}^{b} h(t) n(s)\left[f_{1}\left(s, h_{\text {max }}\right)+f_{2}\left(s, h_{\text {min }}\right)\right] d s \\
& =h(t) \int_{a}^{b} n(s)\left[f_{1}\left(s, h_{\text {max }}\right)+f_{2}\left(s, h_{\text {min }}\right)\right] d s .
\end{aligned}
$$

Let $r_{1}=\int_{a}^{b} m(s)\left[f_{1}\left(s, h_{\min }\right)+f_{2}\left(s, h_{\max }\right)\right] d s, r_{2}=\int_{a}^{b} n(s)\left[f_{1}\left(s, h_{\max }\right)+f_{2}\left(s, h_{\min }\right)\right] d s$. Note that $f=f_{1}+f_{2} \geq 0$ is continuous with $f \not \equiv 0$ and from (3.2), we get $0<r_{1} \leq r_{2}$ and in consequence, $r_{1} h \leq A h+B h \leq r_{2} h$. That is, $A h+B h \in P_{h}$. Hence, all the conditions of Theorem 2.1 are satisfied. It follows from Theorem 2.1 and Corollary 2.5 that the operator equation $A u+B u=\frac{1}{\lambda} u$ has a unique solution $u_{\lambda}^{*}$ in $P_{h}$, that is, $\lambda\left(A u_{\lambda}^{*}+B u_{\lambda}^{*}\right)=u_{\lambda}^{*}$. So $u_{\lambda}^{*}$ is a unique positive solution of the integral equation (3.1) in $P_{h}$ for given $\lambda>0$. From Corollary 2.5, we have (i) if $\varphi_{i}(t)>t^{\frac{1}{2}}(i=1,2)$ for $t \in(0,1)$, then $u_{\lambda}^{*}$ is strictly increasing in $\lambda$, that is, $0<\lambda_{1}<\lambda_{2}$ implies $u_{\lambda_{1}}^{*}<u_{\lambda_{2}}^{*}$; (ii) if there exists $\beta \in(0,1)$ such that $\varphi_{i}(t) \geq t^{\beta}(i=1,2)$ for $t \in(0,1)$, then $u_{\lambda}^{*}$ is continuous in $\lambda$, that is, $\lambda \rightarrow \lambda_{0}\left(\lambda_{0}>0\right)$ implies $\left\|u_{\lambda}^{*}-u_{\lambda_{0}}^{*}\right\| \rightarrow 0$; (iii) if there exists $\beta \in\left(0, \frac{1}{2}\right)$ such that $\varphi_{i}(t) \geq t^{\beta}(i=1,2)$ for $t \in(0,1)$, then $\lim _{\lambda \rightarrow 0^{+}}\left\|u_{\lambda}^{*}\right\|=0$, $\lim _{\lambda \rightarrow+\infty}\left\|u_{\lambda}^{*}\right\|=+\infty$.

Let $A_{\lambda}=\lambda A, B_{\lambda}=\lambda B$. Then $A_{\lambda}, B_{\lambda}$ also satisfy the conditions of Theorem 2.1. By Theorem 2.1, for any initial values $x_{0}, y_{0} \in P_{h}$, constructing successively the sequences

$$
x_{n}=A_{\lambda} x_{n-1}+B_{\lambda} y_{n-1}, \quad y_{n}=A_{\lambda} y_{n-1}+B_{\lambda} x_{n-1}, \quad n=1,2, \ldots,
$$

we have $x_{n} \rightarrow u_{\lambda}^{*}, y_{n} \rightarrow u_{\lambda}^{*}$ as $n \rightarrow+\infty$. That is,

$$
\begin{aligned}
& x_{n}=\lambda \int_{a}^{b} G(t, s)\left[f_{1}\left(s, x_{n-1}(s)\right)+f_{2}\left(s, y_{n-1}(s)\right)\right] d s \rightarrow u_{\lambda}^{*}(t), \\
& y_{n}=\lambda \int_{a}^{b} G(t, s)\left[f_{1}\left(s, y_{n-1}(s)\right)+f_{2}\left(s, x_{n-1}(s)\right)\right] d s \rightarrow u_{\lambda}^{*}(t)
\end{aligned}
$$

as $n \rightarrow+\infty$ 
Theorem 3.2 Assume that $f(t, x)=f_{1}(t, x)+f_{2}(t, x, x) \not \equiv 0$ with $f_{1}(t, x)$ satisfies $\left(\mathrm{H}_{31}\right)$ and

$\left(\mathrm{H}_{33}\right) f_{2}(t, x, y):[a, b] \times[0,+\infty) \times[0,+\infty) \rightarrow[0,+\infty)$ is continuous, increasing in $x \in$ $[0,+\infty)$ for fixed $t \in[a, b], y \in[0,+\infty)$, decreasing in $y \in[0,+\infty)$ for fixed $t \in[a, b]$, $x \in[0,+\infty)$;

$\left(\mathrm{H}_{34}\right)$ for $\eta \in(0,1)$, there exist $\varphi_{i}(\eta) \in(\eta, 1)(i=1,2)$ such that

$$
\begin{aligned}
& f_{1}(t, \eta x) \geq \varphi_{1}(\eta) f_{1}(t, x), \\
& f_{2}\left(t, \eta x, \eta^{-1} y\right) \geq \varphi_{2}(\eta) f_{2}(t, x, y), \quad \forall t \in[a, b], x, y \in[0,+\infty) .
\end{aligned}
$$

Then, for any given $\lambda>0$, the integral equation (3.1) has a unique positive solution $u_{\lambda}^{*}$ in $P_{h}$. Moreover, for any initial values $x_{0}, y_{0} \in P_{h}$, constructing successively the sequences:

$$
\begin{aligned}
& x_{n}=\lambda \int_{a}^{b} G(t, s)\left[f_{1}\left(s, x_{n-1}(s)\right)+f_{2}\left(s, x_{n-1}(s), y_{n-1}(s)\right)\right] d s, \\
& y_{n}=\lambda \int_{a}^{b} G(t, s)\left[f_{1}\left(s, y_{n-1}(s)\right)+f_{2}\left(s, y_{n-1}(s), x_{n-1}(s)\right)\right] d s, \quad n=1,2, \ldots,
\end{aligned}
$$

we have $x_{n} \rightarrow u_{\lambda}^{*}, y_{n} \rightarrow u_{\lambda}^{*}$ as $n \rightarrow+\infty$. Further, the conclusions (i), (ii), and (iii) in Theorem 3.1 also hold.

Proof Define two operators $A: P \rightarrow E$ and $B: P \times P \rightarrow E$ by

$$
A u(t)=\int_{a}^{b} G(t, s) f_{1}(s, u(s)) d s, \quad B(u, v)(t)=\int_{a}^{b} G(t, s) f_{2}(s, u(s), v(s)) d s
$$

It is easy to see that $u$ is the solution of (3.1) if and only if $u=\lambda[A u+B(u, u)]$. From $\left(\mathrm{H}_{31}\right)$ and $\left(\mathrm{H}_{33}\right)$, we know that $A: P \rightarrow P$ is increasing and $B: P \times P \rightarrow P$ is mixed monotone. Further, from $\left(\mathrm{H}_{34}\right)$, we can prove that $A, B$ satisfy $\left(\mathrm{H}_{21}\right)$ and $\left(\mathrm{H}_{22}\right)$. Next we prove that $A h+B(h, h) \in P_{h}$.

For any $t \in[a, b]$, from $\left(\mathrm{H}_{31}\right),\left(\mathrm{H}_{33}\right)$, and (3.2), we have

$$
\begin{aligned}
A h(t)+B(h, h)(t) & =\int_{a}^{b} G(t, s)\left[f_{1}(s, h(s))+f_{2}(s, h(s), h(s))\right] d s \\
& \geq \int_{a}^{b} h(t) m(s)\left[f_{1}\left(s, h_{\min }\right)+f_{2}\left(s, h_{\min }, h_{\max }\right)\right] d s \\
& =h(t) \int_{a}^{b} m(s)\left[f_{1}\left(s, h_{\min }\right)+f_{2}\left(s, h_{\min }, h_{\max }\right)\right] d s, \\
A h(t)+B(h, h)(t) & \leq \int_{a}^{b} h(t) n(s)\left[f_{1}\left(s, h_{\max }\right)+f_{2}\left(s, h_{\max }, h_{\min }\right)\right] d s \\
& =h(t) \int_{a}^{b} n(s)\left[f_{1}\left(s, h_{\max }\right)+f_{2}\left(s, h_{\max }, h_{\min }\right)\right] d s .
\end{aligned}
$$

Let $r_{1}=\int_{a}^{b} m(s)\left[f_{1}\left(s, h_{\min }\right)+f_{2}\left(s, h_{\min }, h_{\max }\right)\right] d s, r_{2}=\int_{a}^{b} n(s)\left[f_{1}\left(s, h_{\max }\right)+f_{2}\left(s, h_{\max }, h_{\min }\right)\right] d s$. Note that $f=f_{1}+f_{2}$ is nonnegative and continuous with $f \neq \equiv$ and from (3.2), we get $0<$ $r_{1} \leq r_{2}$ and in consequence, $r_{1} h \leq A h+B(h, h) \leq r_{2} h$. That is, $A h+B(h, h) \in P_{h}$. Hence, all the conditions of Theorem 2.7 are satisfied. It follows from Theorem 2.7 and Corollary 2.11 
that the operator equation $A u+B(u, u)=\frac{1}{\lambda} u$ has a unique solution $u_{\lambda}^{*}$ in $P_{h}$, that is, $\lambda\left[A u_{\lambda}^{*}+\right.$ $\left.B\left(u_{\lambda}^{*}, u_{\lambda}^{*}\right)\right]=u_{\lambda}^{*}$. So $u_{\lambda}^{*}$ is a unique positive solution of the integral equation (3.1) in $P_{h}$ for given $\lambda>0$. From Corollary 2.11, we have (i) if $\varphi_{i}(t)>t^{\frac{1}{2}}(i=1,2)$ for $t \in(0,1)$, then $u_{\lambda}^{*}$ is strictly increasing in $\lambda$, that is, $0<\lambda_{1}<\lambda_{2}$ implies $u_{\lambda_{1}}^{*}<u_{\lambda_{2}}^{*}$; (ii) if there exists $\beta \in(0,1)$ such that $\varphi_{i}(t) \geq t^{\beta}(i=1,2)$ for $t \in(0,1)$, then $u_{\lambda}^{*}$ is continuous in $\lambda$, that is, $\lambda \rightarrow \lambda_{0}\left(\lambda_{0}>\right.$ 0 ) implies $\left\|u_{\lambda}^{*}-u_{\lambda_{0}}^{*}\right\| \rightarrow 0$; (iii) if there exists $\beta \in\left(0, \frac{1}{2}\right)$ such that $\varphi_{i}(t) \geq t^{\beta}(i=1,2)$ for $t \in(0,1)$, then $\lim _{\lambda \rightarrow 0^{+}}\left\|u_{\lambda}^{*}\right\|=0, \lim _{\lambda \rightarrow+\infty}\left\|u_{\lambda}^{*}\right\|=+\infty$.

Let $A_{\lambda}=\lambda A, B_{\lambda}=\lambda B$. Then $A_{\lambda}, B_{\lambda}$ also satisfy the conditions of Theorem 2.7. By Theorem 2.7, for any initial values $x_{0}, y_{0} \in P_{h}$, constructing successively the sequences $x_{n}=A_{\lambda} x_{n-1}+B_{\lambda}\left(x_{n-1}, y_{n-1}\right), y_{n}=A_{\lambda} y_{n-1}+B_{\lambda}\left(y_{n-1}, x_{n-1}\right), n=1,2, \ldots$, we have $x_{n} \rightarrow u_{\lambda}^{*}$, $y_{n} \rightarrow u_{\lambda}^{*}$ as $n \rightarrow+\infty$. That is,

$$
\begin{aligned}
& x_{n}=\lambda \int_{a}^{b} G(t, s)\left[f_{1}\left(s, x_{n-1}(s)\right)+f_{2}\left(s, x_{n-1}(s), y_{n-1}(s)\right)\right] d s \rightarrow u_{\lambda}^{*}(t), \\
& y_{n}=\lambda \int_{a}^{b} G(t, s)\left[f_{1}\left(s, y_{n-1}(s)\right)+f_{2}\left(s, y_{n-1}(s), x_{n-1}(s)\right)\right] d s \rightarrow u_{\lambda}^{*}(t)
\end{aligned}
$$

as $n \rightarrow+\infty$.

Theorem 3.3 Assume that $f(t, x)=f_{1}(t, x)+f_{2}(t, x, x) \not \equiv 0$ with $f_{1}(t, x)$ satisfies all the conditions of $f_{2}(t, x)$ in Theorem 3.1 and $f_{2}(t, x, y)$ satisfies $\left(\mathrm{H}_{33}\right)$ and $\left(\mathrm{H}_{34}\right)$. Then, for any given $\lambda>0$, the integral equation (3.1) has a unique positive solution $u_{\lambda}^{*}$ in $P_{h}$. Moreover, for any initial values $x_{0}, y_{0} \in P_{h}$, constructing successively the sequences:

$$
\begin{aligned}
& x_{n}=\lambda \int_{a}^{b} G(t, s)\left[f_{1}\left(s, y_{n-1}(s)\right)+f_{2}\left(s, x_{n-1}(s), y_{n-1}(s)\right)\right] d s, \\
& y_{n}=\lambda \int_{a}^{b} G(t, s)\left[f_{1}\left(s, x_{n-1}(s)\right)+f_{2}\left(s, y_{n-1}(s), x_{n-1}(s)\right)\right] d s, \quad n=1,2, \ldots,
\end{aligned}
$$

we have $x_{n} \rightarrow u_{\lambda}^{*}, y_{n} \rightarrow u_{\lambda}^{*}$ as $n \rightarrow+\infty$. Further, the conclusions (i), (ii), and (iii) in Theorem 3.1 also hold.

Proof Similar to the proofs of Theorem 3.1 and Theorem 3.2, the conclusions follow from Theorem 2.12 and Corollary 2.14.

\subsection{Applications to nonlinear elliptic BVPs for the Lane-Emden-Fowler equations}

Let $\Omega$ be a bounded domain with smooth boundary in $\mathbf{R}^{N}(N \geq 1)$. Consider the following singular Dirichlet problem for the Lane-Emden-Fowler equation:

$$
\begin{cases}-\Delta u=\lambda f(x, u), & x \in \Omega, \\ u(x)>0, & x \in \Omega, \\ u(x)=0, & x \in \partial \Omega,\end{cases}
$$

where $\lambda>0$ and the nonlinear term $f(x, u)$ is allowed to be singular on $\partial \Omega$.

The problem (3.3) arises in the study of non-Newtonian fluids, boundary layer phenomena for viscous fluids, chemical heterogeneous catalysts, as well as in the theory of heat conduction in electrically materials (see [28-32]). The theory of singular elliptic boundary value problems for partial differential equations has become an important area of inves- 
tigation in the past three decades, see [28-40] and references therein. By means of subsupersolutions and various techniques related to the maximum principle for elliptic equations, some existence and nonexistence results, a unique positive solution are established. In $[3,7]$, we investigated the existence and uniqueness of positive solutions to the singular Dirichlet problem for the Lane-Emden-Fowler equation (3.3), where $f(x, u)$ is increasing in $u \in(0,+\infty)$ for each $x \in \Omega$ in [3]; $f(t, u)=f_{1}(t, u)+f_{2}(t, u)$ with $f_{i}(t, u)$ is increasing in $u \in(0,+\infty)$ for each $x \in \Omega, i=1,2$ in [7] and $\lambda=1$. However, to our knowledge, the results on the existence-uniqueness of positive solutions for singular elliptic equation are still few. The purpose here is to establish the existence-uniqueness of positive solutions to the singular Dirichlet problem for the Lane-Emden-Fowler equation (3.3) by using some fixed point results in Section 2.

Throughout this subsection, denote $W^{k, l}(\Omega)$ the Sobolev space (see [41]), where $l>1$ and $k$ is a nonnegative integer. And denote $h_{1}$ the eigenfunction corresponding to the smallest eigenvalue $\lambda_{1}$ of the problem $-\Delta \varphi=\lambda \varphi$ in $\Omega$, and $\left.\varphi\right|_{\partial \Omega}=0$. For convenience, we can assume that $h_{1}(x) \geq 0$ in $\bar{\Omega}$. Moreover, it is well known that (see for instance [42]) there exist two positive constants $C_{2}, C_{3}$ such that the first eigenvalue function satisfies

$$
0<C_{2} \leq h_{1}(x)[d(x)]^{-1} \leq C_{3}, \quad x \in \Omega,
$$

where $d(x)=\operatorname{dist}(x, \partial \Omega)$.

Lemma 3.4 (See [43, Theorem 3, p.468]) Let $\Omega$ be a bounded domain in $\mathbf{R}^{N}$ with smooth boundary $\partial \Omega$. Let $u \in L_{\text {loc }}^{1}(\Omega)$ and assume that, for some $k \geq 0$, u satisfies

$$
\begin{cases}-\Delta u+k u \geq 0 & \text { in } \Omega, \\ u \geq 0 & \text { on } \Omega .\end{cases}
$$

Then either $u=0$, or there exists $\varepsilon>0$ such that $u(x) \geq \varepsilon \operatorname{dist}(x, \partial \Omega)$ in $\Omega$.

The proof of this result is due to Brezis and Nirenberg and the result is inspired by the work of Stampachia. Brezis and Nirenberg obtained this result in order to solve a similar eigenvalue problem as considered here. Actually, the result was extended to more general operators, such as $\Delta_{p}$, under some suitable restrictions in order to solve a large class of problems (see for example the problems considered recently in the work of Covei [44]). Here we recall the result since can be used to prove the following simple but useful lemma.

Lemma 3.5 (See [3, Theorem 3.1, p.1278]) Let $\Omega$ be a bounded domain with smooth boundary in $\mathbf{R}^{N}(N \geq 1)$. If $w \in W^{2, l}(\Omega)$ and $w(x)=0$ for $x \in \partial \Omega$, then there exists a constant $M_{1}>0$ such that

$$
|w(x)| \leq M_{1} h_{1}(x), \quad x \in \Omega,
$$

where $M_{1}$ depends only upon $N$ and $\Omega$.

Theorem 3.6 Assume that $f(x, u)=f_{1}(x, u)+f_{2}(x, u)$ and

$\left(\mathrm{H}_{35}\right) f_{i}(x, u), i=1,2$ is nonnegative on $\Omega \times(0,+\infty)$, Hölder continuous in the variable $x$ with the Hölder exponent $\gamma \in(0,1)$ for each $u \in(0,+\infty)$ and continuous in the variable $u$ for each $x \in \Omega$; 
$\left(\mathrm{H}_{36}\right) f_{1}(x, u)$ is increasing in $u$ for each $x$ and $f_{2}(x, u)$ is decreasing in $u$ for each $x$, and for any $t \in(0,1)$, there exists a constant $\varphi_{i}(t) \in(t, 1), i=1,2$, such that

$$
f_{1}(x, t u) \geq \varphi_{1}(t) f_{1}(x, u), \quad f_{2}(x, t u) \leq \frac{1}{\varphi_{2}(t)} f_{2}(x, u) \quad \text { for any } u \in(0,+\infty) ;
$$

$\left(\mathrm{H}_{37}\right) f_{1}(x, u), f_{2}(x, u)$ satisfy the conditions of integrability, i.e.,

$$
\int_{\Omega} f_{i}\left(x, h_{1}(x)\right)^{l} d x<+\infty, \quad i=1,2 \text { for some } l>N .
$$

Then the problem (3.3) has a unique positive solution $u_{\lambda}^{*} \in C^{1, \tau}(\bar{\Omega})$ with respect to $\lambda>0$, where $\tau=1-\frac{N}{l}$. Moreover, (i) if $\varphi_{i}(t)>t^{\frac{1}{2}}(i=1,2)$ for $t \in(0,1)$, then $u_{\lambda}^{*}$ is strictly increasing in $\lambda$, that is, $0<\lambda_{1}<\lambda_{2}$ implies $u_{\lambda_{1}}^{*}<u_{\lambda_{2}}^{*}$; (ii) if there exists $\beta \in(0,1)$ such that $\varphi_{i}(t) \geq t^{\beta}(i=$ 1 ,2) for $t \in(0,1)$, then $u_{\lambda}^{*}$ is continuous in $\lambda$, that is, $\lambda \rightarrow \lambda_{0}\left(\lambda_{0}>0\right)$ implies $\left\|u_{\lambda}^{*}-u_{\lambda_{0}}^{*}\right\| \rightarrow 0$; (iii) if there exists $\beta \in\left(0, \frac{1}{2}\right)$ such that $\varphi_{i}(t) \geq t^{\beta}(i=1,2)$ for $t \in(0,1)$, then $\lim _{\lambda \rightarrow 0^{+}}\left\|u_{\lambda}^{*}\right\|=0$, $\lim _{\lambda \rightarrow+\infty}\left\|u_{\lambda}^{*}\right\|=+\infty$.

Proof For the sake of convenience, set $E=C(\bar{\Omega})$, the Banach space of continuous functions on $\bar{\Omega}$ with the norm $\|u\|=\max \{|u(x)|: x \in \bar{\Omega}\}$. Set $P=\{u \in C(\bar{\Omega}) \mid u(x) \geq 0, x \in \bar{\Omega}\}$, the standard cone. It is clear that $P$ is a normal cone in $E$ and the normality constant is $1, P_{h_{1}}$ is given as in the Section 1.1. We divide the proof into several steps.

Step 1. We consider the following linear elliptic boundary value problem:

$$
\begin{cases}-\Delta w=f_{1}(x, u), & x \in \Omega, \\ w(x)>0, & x \in \Omega, \\ w(x)=0, & x \in \partial \Omega\end{cases}
$$

where $u \in P_{h_{1}}$. Since $u \in P_{h_{1}}$, we can choose a sufficiently small number $r_{0} \in(0,1)$ such that

$$
r_{0} h_{1}(x) \leq u(x) \leq \frac{1}{r_{0}} h_{1}(x), \quad x \in \bar{\Omega} .
$$

Then from $\left(\mathrm{H}_{36}\right)$,

$$
\begin{aligned}
& f_{1}(x, u(x)) \geq f_{1}\left(x, r_{0} h_{1}(x)\right) \geq \varphi_{1}\left(r_{0}\right) f_{1}\left(x, h_{1}(x)\right), \quad x \in \Omega, \\
& f_{1}(x, u(x)) \leq f_{1}\left(x, \frac{1}{r_{0}} h_{1}(x)\right) \leq \frac{1}{\varphi_{1}\left(r_{0}\right)} f_{1}\left(x, h_{1}(x)\right), \quad x \in \Omega .
\end{aligned}
$$

Thus we get by applying the integrability condition $\left(\mathrm{H}_{37}\right)$ that says that

$$
\int_{\Omega}\left[f_{1}(x, u(x))\right]^{l} d x<+\infty
$$

namely, $f_{1}(x, u) \in L^{l}(\Omega)$. By the classical theory of linear elliptic equations (see [45]), the problem (3.5) admits a unique strong solution $w_{u} \in W^{2, l}(\Omega) \cap W_{0}^{1, l}(\Omega)$. Recall that $l>N$. Using the Sobolev imbedding theory, $w_{u} \in C^{1, \tau}(\bar{\Omega})$ with $\tau=1-\frac{N}{l}$. Now we define an operator $A: P_{h_{1}} \rightarrow E$ by

$$
A u(x)=w_{u}(x), \quad u \in P_{h_{1}},
$$


where $w_{u}$ is the unique strong solution of (3.5) for $u \in P_{h_{1}}$. Evidently, $A: P_{h_{1}} \rightarrow P$. Suppose that $\phi$ is the solution of (3.5) with $u=h_{1}$, then $A h_{1}=\phi \in C^{1, \tau}(\bar{\Omega})$. Then from Lemma 3.5, there exists a positive constant $C_{\phi}$ such that

$$
\phi(x) \leq C_{\phi} h_{1}(x), \quad x \in \bar{\Omega} .
$$

Note that $f\left(x, h_{1}(x)\right) \geq 0$. By the maximal principle, $\phi(x) \geq 0$. Since $\phi(x)>0$ for $x \in \Omega$, an application of Lemma 3.4 implies that

$$
\phi(x) \geq \varepsilon d(x), \quad x \in \bar{\Omega} .
$$

Combining (3.4) and (3.9), there exists a positive constant $c_{\phi}$ such that

$$
\phi(x) \geq c_{\phi} h_{1}(x), \quad x \in \bar{\Omega} .
$$

Let $w_{u}$ is the unique strong solution of (3.5) for $u \in P_{h_{1}}$. From (3.6) and (3.7), and applying the comparison principle, we conclude that

$$
\varphi_{1}\left(r_{0}\right) \phi(x) \leq w_{u}(x) \leq \frac{1}{\varphi_{1}\left(r_{0}\right)} \phi(x), \quad x \in \bar{\Omega},
$$

and, from (3.8) and (3.10), we get $A u=w_{u} \in P_{h_{1}}$ for any $u \in P_{h_{1}}$. So we find that $A: P_{h_{1}} \rightarrow$ $P_{h_{1}}$ is well defined. Further, from $\left(\mathrm{H}_{36}\right)$ and the comparison principle, we can easily prove that $A: P_{h_{1}} \rightarrow P_{h_{1}}$ is increasing. In the following we prove that $A(t u) \geq \varphi_{1}(t) A u$ for any $u \in P_{h_{1}}$ and $t \in(0,1)$. For any $u \in P_{h_{1}}$ and $t \in(0,1)$, we have

$$
\begin{cases}-\Delta A(t u)=f_{1}(x, t u), & x \in \Omega, \\ A(t u)(x)=0, & x \in \partial \Omega\end{cases}
$$

and

$$
\begin{cases}-\Delta \varphi_{1}(t) A u=\varphi_{1}(t) f_{1}(x, u), & x \in \Omega, \\ \varphi_{1}(t) A u(x)=0, & x \in \partial \Omega .\end{cases}
$$

From $\left(\mathrm{H}_{36}\right)$ we also get $f_{1}(x, t u(x))-\varphi_{1}(t) f_{2}(x, u(x)) \geq 0$ for any $x \in \bar{\Omega}$. Therefore,

$$
\begin{cases}-\Delta\left(A(t u)-\varphi_{1}(t) A u\right) \geq 0, & x \in \Omega, \\ A(t u)(x)-\varphi_{1}(t) A u(x)=0, & x \in \partial \Omega .\end{cases}
$$

Using the comparison principle again, we can obtain $A(t u) \geq \varphi_{1}(t) A u$ immediately. So we have $A(t u) \geq \varphi_{1}(t) A u$ for $t \in(0,1), u \in P_{h_{1}}$.

Step 2. We consider the following linear elliptic boundary value problem:

$$
\begin{cases}-\Delta w=f_{2}(x, u), & x \in \Omega, \\ w(x)>0, & x \in \Omega, \\ w(x)=0, & x \in \partial \Omega,\end{cases}
$$

where $u \in P_{h_{1}}$. Since $u \in P_{h_{1}}$, we can choose a sufficiently small number $r_{0} \in(0,1)$ such that

$$
r_{0} h_{1}(x) \leq u(x) \leq \frac{1}{r_{0}} h_{1}(x), \quad x \in \bar{\Omega} .
$$


Then from $\left(\mathrm{H}_{36}\right)$,

$$
\begin{aligned}
& f_{2}(x, u(x)) \leq f_{2}\left(x, r_{0} h_{1}(x)\right) \leq \frac{1}{\varphi_{2}\left(r_{0}\right)} f_{2}\left(x, h_{1}(x)\right), \quad x \in \Omega, \\
& f_{2}(x, u(x)) \geq f_{2}\left(x, \frac{1}{r_{0}} h_{1}(x)\right) \geq \varphi_{2}\left(r_{0}\right) f_{2}\left(x, h_{1}(x)\right), \quad x \in \Omega .
\end{aligned}
$$

Thus we get by applying the integrability condition $\left(\mathrm{H}_{37}\right)$ that says that $\int_{\Omega}\left[f_{2}(x, u(x))\right]^{l} d x<$ $+\infty$, namely, $f_{2}(x, u) \in L^{l}(\Omega)$. By the classical theory of linear elliptic equations, the problem (3.11) admits a unique strong solution $w_{u} \in W^{2, l}(\Omega) \cap W_{0}^{1, l}(\Omega)$. Recall that $l>N$. Using the Sobolev imbedding theory, $w_{u} \in C^{1, \tau}(\bar{\Omega})$ with $\tau=1-\frac{N}{l}$. Now we define an operator $B: P_{h_{1}} \rightarrow E$ by $B u(x)=w_{u}(x), u \in P_{h_{1}}$, where $w_{u}$ is the unique strong solution of (3.11) for $u \in P_{h_{1}}$. Similar to Step 1,we can prove that $B: P_{h_{1}} \rightarrow P_{h_{1}}$ is well defined. Using the comparison principle again, we can easily see that $B: P_{h_{1}} \rightarrow P_{h_{1}}$ is decreasing and $B(t u) \leq \frac{1}{\varphi_{2}(t)} B u$ for any $u \in P_{h_{1}}$ and $t \in(0,1)$.

Step 3. Now all the conditions of Corollary 2.2 are satisfied. It follows from Corollary 2.2 and Corollary 2.5 that the operator equation $A u+B u=\frac{1}{\lambda} u$ has a unique solution $u_{\lambda}^{*}$ in $P_{h_{1}}$, that is, $\lambda\left(A u_{\lambda}^{*}+B u_{\lambda}^{*}\right)=u_{\lambda}^{*}$. So $u_{\lambda}^{*}$ is a unique positive solution of the problem (3.3) in $P_{h_{1}}$ for given $\lambda>0$. By the theory of the linear elliptic equation, the problem

$$
\begin{cases}-\Delta w=\lambda\left[f_{1}\left(x, u_{\lambda}^{*}\right)+f_{2}\left(x, u_{\lambda}^{*}\right)\right], & x \in \Omega, \\ w(x)>0, & x \in \Omega, \\ w(x)=0, & x \in \partial \Omega,\end{cases}
$$

admits a unique solution $\overline{u_{\lambda}^{*}} \in W^{2, l}(\Omega) \cap W_{0}^{1, l}(\Omega)$, and hence $\overline{u_{\lambda}^{*}} \in C^{1, \tau}(\Omega)$. Recalling the uniqueness of the solution of (3.3), one can see easily that $\overline{u_{\lambda}^{*}}=u_{\lambda}^{*}$. Thus the problem (3.3) has a unique classical solution $u_{\lambda}^{*} \in C^{1, \tau}(\bar{\Omega})$. Moreover, by using Corollary 2.5 and the theory of the linear elliptic equation, we can easily prove that (i) if $\varphi_{i}(t)>t^{\frac{1}{2}}(i=1,2)$ for $t \in(0,1)$, then $u_{\lambda}^{*}$ is strictly increasing in $\lambda$, that is, $0<\lambda_{1}<\lambda_{2}$ implies $u_{\lambda_{1}}^{*}<u_{\lambda_{2}}^{*}$; (ii) if there exists $\beta \in(0,1)$ such that $\varphi_{i}(t) \geq t^{\beta}(i=1,2)$ for $t \in(0,1)$, then $u_{\lambda}^{*}$ is continuous in $\lambda$, that is, $\lambda \rightarrow \lambda_{0}\left(\lambda_{0}>0\right)$ implies $\left\|u_{\lambda}^{*}-u_{\lambda_{0}}^{*}\right\| \rightarrow 0$; (iii) if there exists $\beta \in\left(0, \frac{1}{2}\right)$ such that $\varphi_{i}(t) \geq t^{\beta}$ $(i=1,2)$ for $t \in(0,1)$, then $\lim _{\lambda \rightarrow 0^{+}}\left\|u_{\lambda}^{*}\right\|=0, \lim _{\lambda \rightarrow+\infty}\left\|u_{\lambda}^{*}\right\|=+\infty$.

Similar to the proofs of Theorem 3.6 and Theorems 3.2, 3.3, we can easily obtain the following conclusions.

Theorem 3.7 Assume that $f(x, u)=f_{1}(x, u)+f_{2}(x, u, u)$ and $f_{1}(x, u)$ satisfies all the conditions of Theorem 3.6, $f_{2}$ satisfies

$\left(\mathrm{H}_{38}\right) f_{2}(x, u, v)$ is nonnegative on $\Omega \times(0,+\infty) \times(0,+\infty)$, Hölder continuous in the variable $x$ with the Hölder exponent $\gamma \in(0,1)$ for each $u, v \in(0,+\infty)$ and is continuous in the variables $u, v$ for each $x \in \Omega$;

$\left(\mathrm{H}_{39}\right)$ for any $t \in(0,1)$, there exists a constant $\varphi_{2}(t) \in(t, 1)$ such that

$$
f_{2}\left(x, t u, t^{-1} v\right) \geq \varphi_{2}(t) f_{2}(x, u, v) \quad \text { for any } u, v \in(0,+\infty) \text {; }
$$

$\left(\mathrm{H}_{310}\right) f_{2}(x, u, v)$ satisfies the condition of integrability, i.e.,

$$
\int_{\Omega} f_{2}\left(x, h_{1}(x), h_{1}(x)\right)^{l} d x<+\infty \text { for some } l>N .
$$


Then the problem (3.3) has a unique positive solution $u_{\lambda}^{*} \in C^{1, \tau}(\bar{\Omega})$ with respect to $\lambda>0$, where $\tau=1-\frac{N}{l}$. Further, the conclusions (i), (ii), and (iii) in Theorem 3.6 also hold.

Theorem 3.8 Assume that $f(x, u)=f_{1}(x, u)+f_{2}(x, u, u)$ with $f_{1}$ satisfying all the conditions of $f_{2}$ in Theorem 3.6 and $f_{2}$ satisfying $\left(\mathrm{H}_{38}\right),\left(\mathrm{H}_{39}\right)$, and $\left(\mathrm{H}_{310}\right)$. Then the problem (3.3) has a unique positive solution $u_{\lambda}^{*} \in C^{1, \tau}(\bar{\Omega})$ with respect to $\lambda>0$, where $\tau=1-\frac{N}{l}$. Further, the conclusions (i), (ii), and (iii) in Theorem 3.6 also hold.

\section{Competing interests}

The authors declare that they have no competing interests.

\section{Authors' contributions}

The authors declare that the study was realized in collaboration with the same responsibility. All authors read and approved the final manuscript.

\section{Author details}

${ }^{1}$ Basic Course Department, Business College of Shanxi University, Taiyuan, Shanxi 030031, P.R. China. ${ }^{2}$ School of Mathematical Sciences, Shanxi University, Taiyuan, Shanxi 030006, P.R. China.

\section{Acknowledgements}

The research was supported by the Youth Science Foundation of China (11201272), the Science Foundations of Shanxi Province (2010021002-1; 2013011003-3) and the Science Foundation of Business College of Shanxi University (2012050).

Received: 12 September 2013 Accepted: 20 January 2014 Published: 10 Feb 2014

\section{References}

1. Agarwal, RP, O'Regan, D: A multiplicity result for second order impulsive differential equations via the Leggett Williams fixed point theorem. Appl. Math. Comput. 161, 433-439 (2005)

2. Avery, Rl, Henderson, J: Three symmetric positive solutions for a second order boundary value problem. Appl. Math. Lett. 13(3), 1-7 (2000)

3. Guo, CM, Zhai, CB, Song, RP: An existence and uniqueness result for the singular Lane-Emden-Fowler equation. Nonlinear Anal. 72, 1275-1279 (2010)

4. Karaca, IY: Nonlinear triple-point problems with change of sign. Comput. Math. Appl. 55(14), 691-703 (2008)

5. Yang, C, Zhai, CB, Yan, JR: Positive solutions of three-point boundary value problem for second order differential equations with an advanced argument. Nonlinear Anal. 65, 2013-2023 (2006)

6. Yang, C, Zhai, CB: Uniqueness of positive solutions for a fractional differential equation via a fixed point theorem of a sum operator. Electron. J. Differ. Equ. 70, 1-8 (2012)

7. Zhai, CB, Anderson, DR: A sum operator equation and applications to nonlinear elastic beam equations and Lane-Emden-Fowler equations. J. Math. Anal. Appl. 375, 388-400 (2011)

8. Zhai, CB, Song, RP, Han, QQ: The existence and the uniqueness of symmetric positive solutions for a fourth-order boundary value problem. Comput. Math. Appl. 62, 2639-2647 (2011)

9. Zhai, CB, Yan, WP, Yang, C: A sum operator method for the existence and uniqueness of positive solutions to Riemann-Liouville fractional differential equation boundary value problems. Commun. Nonlinear Sci. Numer. Simul. 18, 858-866 (2013)

10. Zhai, CB, Zhang, LL: New fixed point theorems for mixed monotone operators and local existence-uniqueness of positive solutions for nonlinear boundary value problems. J. Math. Anal. Appl. 382, 594-614 (2011)

11. Zhang, JM, Zhai, CB: Existence and uniqueness results for perturbed Neumann boundary value problems. Bound. Value Probl. 2010, Article ID 494210 (2010)

12. Zhang, LL, Zhai, CB: Existence and uniqueness results for three-point boundary value problems. In: Proceedings of the 30th Chinese Control Conference, Yantai, China, pp. 460-463 (2011)

13. Amann, H: Fixed point equations and nonlinear eigenvalue problems in ordered Banach spaces. SIAM Rev. 18(4), 620-709 (1976)

14. Avery, RI, Peterson, AC: Three positive fixed points of nonlinear operators on ordered Banach spaces. Comput. Math. Appl. 42, 313-322 (2001)

15. Chen, YZ: Stability of positive fixed points of nonlinear operators. Positivity 6, 47-57 (2002)

16. Ilic, D, Rakocevic, V: Common fixed points for maps on cone metric space. J. Math. Anal. Appl. 341, 876-882 (2008)

17. Jachymski, J: The contraction principle for mappings on a metric space with a graph. Proc. Am. Math. Soc. 136, 1359-1373 (2008)

18. Li, K, Liang, J, Xiao, TJ: A fixed point theorem for convex and decreasing operators. Nonlinear Anal. 63, e209-e206 (2005)

19. Nieto, JJ, Pouso, RL, Rodriguez-Lopez, R: Fixed point theorems in ordered abstract spaces. Proc. Am. Math. Soc. 135 2505-2517 (2007)

20. Zhai, CB, Guo, CM: On $\alpha$-convex operators. J. Math. Anal. Appl. 316, 556-565 (2006)

21. Zhai, CB, Hao, MR: Fixed point theorems for mixed monotone operators with perturbation and applications to fractional differential equation boundary value problems. Nonlinear Anal. 75, 2542-2551 (2012) 
22. Zhai, CB, Cao, XM: Fixed point theorems for $\tau-\varphi$-concave operators and applications. Comput. Math. Appl. 59, 532-538 (2010)

23. Zhao, Z: Multiple fixed points of a sum operator and applications. J. Math. Anal. Appl. 360, 1-6 (2009)

24. Sang, YB, Wei, ZL, Dong, W: The existence of multiple fixed points for the sum of two operators and applications. Fixed Point Theory 13(1), 193-204 (2012)

25. Deimling, K: Nonlinear Functional Analysis. Springer, Berlin (1985)

26. Guo, D, Lakshmikantham, V: Nonlinear Problems in Abstract Cones. Academic Press, Boston (1988)

27. Guo, D: Existence and uniqueness of positive fixed points for mixed monotone operators and applications. Appl. Anal. 46, 91-100 (1992)

28. Crandall, MG, Rabinowitz, PH, Tartar, L: On a Dirichlet problem with a singular nonlinearity. Commun. Partial Differ Equ. 2(2), 193-222 (1977)

29. Fulks, W, Maybee, JS: A singular nonlinear elliptic equation. Osaka J. Math. 12, 1-19 (1960)

30. Ghergu, M, Rădulescu, V: Sublinear singular elliptic problems with two parameters. J. Differ. Equ. 195, $520-536$ (2003)

31. Ghergu, M, Rădulescu, V: Ground state solutions for the singular Lane-Emden-Fowler equation with sublinear convection term. J. Math. Anal. Appl. 333, 265-273 (2007)

32. Stuart, CA: Existence and approximation of solutions of nonlinear elliptic equations. Math. Z. 147, 53-63 (1976)

33. Coclite, MM, Palmieri, G: On a singular nonlinear Dirichlet problem. Commun. Partial Differ. Equ. 14, 1315-1327 (1989)

34. Dupaigne, L, Ghergu, M, Rădulescu, V: Lane-Emden-Fowler equations with convection and singular potential. J. Math Pures Appl. 87, 563-581 (2007)

35. Ghergu, M, Rădulescu, V: Singular Elliptic Problems. Bifurcation and Asymptotic Analysis. Oxford Lecture Series in Mathematics and Its Applications, vol. 37. Oxford University Press, London (2008)

36. Hernández, J, Mancebo, F, Vega, JM: Positive solutions for singular nonlinear elliptic equations. Proc. R. Soc. Edinb. A $137,41-62$ (2007)

37. Kristály, A, Rădulescu, V Varga, C. Variational Principles in Mathematical Physics, Geometry, and Economics: Qualitative Analysis of Nonlinear Equations and Unilateral Problems. Encyclopedia of Mathematics and Its Applications, vol. 136. Cambridge University Press, Cambridge (2010)

38. Lazer, AC, Mckenna, PJ: On a singular nonlinear elliptic boundary value problem. Proc. Am. Math. Soc. 111, 721-730 (1991)

39. Shi, J, Yao, M: On a singular nonlinear semilinear elliptic problem. Proc. R. Soc. Edinb., Sect. A, Math. 128, 1389-1401 (1998)

40. Zhang, ZJ: The asymptotic behaviour of the unique solution for the singular Lane-Emden-Fowler equation. J. Math Anal. Appl. 312, 33-43 (2005)

41. Adams, RA: Sobolev Spaces. Academic Press, New York (1975)

42. Wiegner, M: A degenerate diffusion equation with a nonlinear source term. Nonlinear Anal. 28(12), 1977-1995 (1997)

43. Brezis, $H$, Nirenberg, L: Minima locaux relatifs a $C^{1}$ et $H^{1}$. C. R. Acad. Sci. Paris 317, 465-472 (1993)

44. Covei, DP: Quasilinear problems with the competition between convex and concave nonlinearities and variable potentials. Int. J. Math. 24(1), Article ID 1350005 (2013)

45. Ladyzhenskaya, OA, Ural'ceva, NN: Linear and Quasilinear Elliptic Equations. Academic Press, New York (1968) (English transl.)

10.1186/1029-242X-2014-58

Cite this article as: Yang et al.: Uniqueness of positive solutions for several classes of sum operator equations and applications. Journal of Inequalities and Applications 2014, 2014:58

\section{Submit your manuscript to a SpringerOpen ${ }^{\odot}$ journal and benefit from:}

- Convenient online submission

- Rigorous peer review

- Immediate publication on acceptance

- Open access: articles freely available online

- High visibility within the field

- Retaining the copyright to your article 